\title{
A Revised Scheme for the WRF Surface Layer Formulation
}

\author{
Pedro A. JimÉnez,* Jimy Dudhia, ${ }^{+}$J. Fidel GonZÁlez-Rouco, ${ }^{\#}$ Jorge Navarro, ${ }^{@}$ \\ Juan P. Montávez, ${ }^{\&}$ And Elena García-Bustamante*** \\ * División de Energías Renovables, CIEMAT, Madrid, Spain, and Mesoscale and Microscale Meteorology Division, \\ NCAR, Boulder, Colorado \\ ${ }^{+}$Mesoscale and Microscale Meteorology Division, NCAR, Boulder, Colorado \\ \# Departamento de Astrofísica y Ciencias de la Atmósfera, Universidad Complutense de Madrid, Madrid, Spain \\ @ División de Energías Renovables, CIEMAT, Madrid, Spain \\ \& Departamento de Física, Universidad de Murcia, Murcia, Spain \\ ** Departamento de Astrofísica y Ciencias de la Atmósfera, Universidad Complutense de Madrid, and División \\ de Energías Renovables, CIEMAT, Madrid, Spain
}

(Manuscript received 24 February 2011, in final form 17 August 2011)

\begin{abstract}
This study summarizes the revision performed on the surface layer formulation of the Weather Research and Forecasting (WRF) model. A first set of modifications are introduced to provide more suitable similarity functions to simulate the surface layer evolution under strong stable/unstable conditions. A second set of changes are incorporated to reduce or suppress the limits that are imposed on certain variables in order to avoid undesired effects (e.g., a lower limit in $u_{*}$ ). The changes introduced lead to a more consistent surface layer formulation that covers the full range of atmospheric stabilities. The turbulent fluxes are more (less) efficient during the day (night) in the revised scheme and produce a sharper afternoon transition that shows the largest impacts in the planetary boundary layer meteorological variables. The most important impacts in the near-surface diagnostic variables are analyzed and compared with observations from a mesoscale network.
\end{abstract}

\section{Introduction}

The lowest part of the planetary boundary layer (PBL) wherein the turbulent fluxes vary less than $10 \%$ of their magnitude is known as the atmospheric surface layer (Stull 1988). Meteorological variables experience a sharp variation with height within this layer that exhibits the most significant exchanges of momentum, heat, and moisture (Arya 1988). The surface layer state determines the land-atmosphere interaction and, thus, its accurate formulation is crucial to provide an adequate atmospheric evolution by numerical models.

The Monin-Obukhov similarity theory (Obukhov 1946; Monin and Obukhov 1954) is a widely used framework to compute the surface turbulent fluxes (Beljaars and Holtslag 1991). The theory also provides information of the profiles within the surface layer that are used to diagnose meteorological variables at their typical

\footnotetext{
Corresponding author address: Pedro A. Jimenez, Mesoscale and Microscale Meteorology Division, National Center for Atmospheric Research, 3450 Mitchell Ln., Boulder, CO 80301.

E-mail: jimenez@ucar.edu
}

observational height such as the wind at $10 \mathrm{~m}$ or the temperature and moisture at $2 \mathrm{~m}$. A limitation, however, is that the predicted similarity functions $\left(\phi_{h, m}\right)$ necessary to compute both the fluxes and the profiles need to be determined empirically.

The Kansas field program (Izumi 1971) provided estimations of the similarity functions for a limited range of atmospheric stabilities (Businger et al. 1971; Dyer 1974; Hicks 1976). For this reason, extensions to highly stable situations (e.g., Webb 1970; van Ulden and Holtslag 1985; Holtslag and de Bruin 1988; Beljaars and Holtslag 1991; Cheng and Brutsaert 2005) as well as highly unstable conditions (e.g., Brutsaert 1992; Fairall et al. 1996; Grachev et al. 2000; Wilson 2001; Fairall et al. 2003) have been proposed. For instance, Fairall et al. (1996, hereafter F96) used the asymptotic behavior predicted by the theory to extend the Kansas type of similarity functions to higher instabilities. The proposed similarity functions are therefore valid from neutral to free convective situations. Similarly, Cheng and Brutsaert (2005, hereafter CB05) found an asymptotic behavior of the similarity functions for the stable part and derived functions valid from neutral situations to very stable conditions. 
Hence, combining similarity functions such as the ones proposed by F96 and CB05 allows one to cover more accurately the full range of atmospheric stabilities by the Monin-Obukhov similarity theory.

The purpose of this investigation is to improve the surface layer formulation of the Weather Research and Forecast model (WRF; Skamarock et al. 2008), in particular, the surface layer scheme based on the fifthgeneration Pennsylvania State University-National Center for Atmospheric Research Mesoscale Model (MM5) parameterization (Grell et al. 1994). Although the scheme is widely used for quite different atmospheric investigations (e.g., Weisman et al. 2008; Jingyong et al. 2008; Jiménez et al. 2010a), it uses Kansas-type similarity functions with their limited coverage of atmospheric stabilities. Here, the similarity functions are replaced by those proposed by F96 and CB05 in order to provide the scheme with a more appropriate framework for strongly stable/unstable conditions.

An additional target of this investigation is to review the limits that are imposed to certain variables (e.g., $u_{*}$, the friction velocity) in order to prevent undesirable effects from the formulation. It has been found herein that these limits can be reduced or removed in order to provide a less restrictive, and more consistent, surface layer formulation that covers the full range of atmospheric stabilities.

The impact that these changes produce in the surface fluxes, the diagnostic surface meteorological variables, and the PBL dynamics is analyzed. The most important impacts in the near-surface variables have been tested against observations from a mesoscale network located in the northeast of the Iberian Peninsula (Jiménez et al. 2010b). The observations have been used in previous studies with quite different orientations (e.g., Jiménez et al. 2009a; García-Bustamante et al. 2011). A complete summer season has been simulated herein (at a high horizontal resolution of $2 \mathrm{~km}$ ) in order to obtain a statistically robust characterization of the changes introduced by the new formulation. The standard WRF model output has been complemented by recording the surface layer variables every time step at the observational sites in order to provide a detailed evolution of the atmosphere within this layer.

\section{Surface layer parameterization}

This section describes the current WRF surface layer formulation (surface layer physics option 1 in WRF, section 2a), its limitations (section 2b), and the revised formulation here proposed in order to overcome the current problems (section 2c).

The computation of the fluxes in WRF not only depends on the surface layer physics but also in the land surface model. The present description of fluxes follows the definition of the soil scheme used in this investigation (surface-physics option 1 in WRF; Dudhia 1996; Dudhia et al. 2004), but the changes described apply equally to other land surface options. More details of the WRF configuration used in this investigation will be provided in section 3 .

\section{a. WRF surface layer}

The surface layer is assumed to be the first vertical layer and the surface fluxes are parameterized as follows:

$$
\begin{gathered}
\tau=\rho u_{*}^{2}=\rho C_{d} U^{2}, \\
H=-\rho c_{p} u_{*} \theta_{*}=-\rho c_{p} C_{h} U\left(\theta_{a}-\theta_{g}\right), \\
\mathrm{LH}=L_{e} \rho u_{*} q_{*}=L_{e} \rho M C_{q} U\left(q_{g}-q_{a}\right),
\end{gathered}
$$

where $\tau, H$, and LH are the fluxes of momentum, sensible heat, and latent heat, respectively; $\theta_{*}$ and $q_{*}$ are the temperature and moisture scales, respectively; $\rho$ is the air density in the surface layer; $c_{p}$ is the specific heat capacity at constant pressure; and $U$ is the wind speed in the lower layer enhanced by a convective velocity following Beljaars (1995) and a subgrid velocity following Mahrt and Sun (1995). This last correction only applies for horizontal grid resolutions higher than $5 \mathrm{~km}$. Here $L_{e}$ is the latent heat of vaporization; $M$ is the soil moisture availability; $\theta_{a}$ and $\theta_{g}$ are the air and ground surface potential temperature, respectively; $q_{g}$ is the saturated specific humidity at the ground; $q_{a}$ is the specific humidity in the surface layer; and $C_{d}, C_{h}$, and $C_{q}$ are the dimensionless bulk transfer coefficients (Stull 1988) for momentum, heat, and moisture, respectively.

The Monin-Obukhov similarity theory is used to calculate the transfer coefficients. The dimensionless wind shear and potential temperature gradient are usually expressed as (e.g., Arya 1988)

$$
\frac{k z}{u_{*}} \frac{\partial u_{a}}{\partial z}=\phi_{m}\left(\frac{z}{L}\right) ; \quad \frac{k z}{\theta_{*}} \frac{\partial \theta}{\partial z}=\phi_{h}\left(\frac{z}{L}\right),
$$

where $k=0.4$ is the von Kármán constant, $u_{a}$ is the wind speed at level $z$, and $L$ is the Obukhov length (Obukhov 1946). Integrating the equations with respect to height $z$, leads to

$$
\begin{aligned}
u_{a} & =\frac{u_{*}}{k}\left[\ln \left(\frac{z}{z_{0}}\right)-\psi_{m}\left(\frac{z}{L}\right)+\psi_{m}\left(\frac{z_{0}}{L}\right)\right], \\
\left(\theta_{a}-\theta_{g}\right) & =\frac{\theta_{*}}{k}\left[\ln \left(\frac{z}{z_{0}}\right)-\psi_{h}\left(\frac{z}{L}\right)+\psi_{h}\left(\frac{z_{0}}{L}\right)\right],
\end{aligned}
$$


where $z_{0}$ is the roughness length and $\psi_{m, h}$ are the integrated similarity functions for momentum and heat that are defined as follows (e.g., Panofsky 1963):

$$
\psi_{m, h}\left(\frac{z}{L}\right) \equiv \int_{0}^{z / L}\left[1-\phi_{m, h}(\zeta)\right] \frac{d \zeta}{\zeta} .
$$

Combining Eq. (1) and Eq. (4) and neglecting the contribution of $\psi_{m}\left(z_{0} / L\right)$ allows one to obtain the bulk transfer coefficient for momentum:

$$
C_{d}=\frac{k^{2}}{\left[\ln \left(\frac{z}{z_{0}}\right)-\psi_{m}\left(\frac{z}{L}\right)\right]^{2}}
$$

Analogously, combining Eq. (2) with Eqs. (4) and (5), and neglecting the contribution of $\psi_{h}\left(z_{0} / L\right)$ allows one to obtain the bulk transfer coefficient for heat:

$$
C_{h}=\frac{k^{2}}{\left[\ln \left(\frac{z}{z_{0}}\right)-\psi_{m}\left(\frac{z}{L}\right)\right]\left[\ln \left(\frac{z}{z_{0}}\right)-\psi_{h}\left(\frac{z}{L}\right)\right]}
$$

where it has been assumed that $u_{a}=U$.

For the case of moisture the surface layer formulation follows Carlson and Boland (1978). The existence is assumed of a viscous sublayer from the ground to a height $z_{l}\left(z_{l}=0.01 \mathrm{~m}\right.$ over land and $z_{0}$ over water), and a turbulent layer wherein Monin-Obukhov theory is applicable from $z_{l}$ to $z$. A similar derivation to the one used to obtain the transfer coefficients for momentum and heat leads to obtain the value of the bulk transfer coefficient for moisture (Carlson and Boland 1978; Grell et al. 1994):

$$
C_{q}=\frac{k^{2}}{\left[\ln \left(\frac{z}{z_{0}}\right)-\psi_{m}\left(\frac{z}{L}\right)\right]\left[\ln \left(\frac{\rho c_{p} k u_{*} z}{c_{s}}+\frac{z}{z_{l}}\right)-\psi_{h}\left(\frac{z}{L}\right)\right]},
$$

where $c_{s}$ is the effective heat transfer coefficient for nonturbulent processes. Note that it has been assumed that the dimensionless similarity function for moisture is the same as heat. This hypothesis is based on experimental evidence (e.g., Dyer 1967; Dyer and Bradley 1982) but it has been recently questioned (e.g., Park et al. 2009).

The integrated similarity functions are calculated according to four stability regimes (Zhang and Anthes 1982) defined in terms of the bulk Richardson number:

$$
\mathrm{Ri}_{b}=\frac{g}{\theta_{a}} z \frac{\theta_{\mathrm{va}}-\theta_{\mathrm{vg}}}{U^{2}}
$$

where $g$ is the gravitational acceleration, $\theta_{\mathrm{va}}$ is the virtual potential temperature of the air in the surface layer, and $\theta_{\mathrm{vg}}$ is the virtual potential temperature of the ground. To prevent $\mathrm{Ri}_{b}$ from being inordinately high, a lower limit of $0.1 \mathrm{~m} \mathrm{~s}^{-1}$ is applied to $U$.

The first regime, $\mathrm{Ri}_{b} \geq 0.2$, is associated with stable (nighttime) conditions and

$$
\psi_{m}=\psi_{h}=-10 \ln \left(\frac{z}{z_{0}}\right)
$$

The second one, $0<\mathrm{Ri}_{b}<0.2$, corresponds with a damped mechanical turbulence regime wherein

$$
\psi_{m}=\psi_{h}=-5 \mathrm{Ri}_{b} \frac{\ln \left(\frac{z}{z_{0}}\right)}{1.1-5 \mathrm{Ri}_{b}} .
$$

The third regime, $\mathrm{Ri}_{b}=0$, is associated with forced convection:

$$
\psi_{m}=\psi_{h}=0
$$

and the fourth one, $\mathrm{Ri}_{b}<0$, with free convection:

$$
\begin{aligned}
& \psi_{m}=2 \ln \left(\frac{1+x}{2}\right)+\ln \left(\frac{1+x^{2}}{2}\right)-2 \tan ^{-1} x+\frac{\pi}{2} \\
& \psi_{h}=2 \ln \left(\frac{1+x^{2}}{2}\right)
\end{aligned}
$$

wherein $x=[1-16(z / L)]^{1 / 4}$ and the Monin-Obukhov stability parameter:

$$
\frac{z}{L}=k \frac{g}{\theta_{a}} z \frac{\theta_{*}}{u_{*}^{2}}
$$

is calculated using the friction velocity, $u_{*}=k U /[\ln (z)$ $\left.\left.z_{0}\right)-\psi_{m}(z / L)\right]$, and the temperature scale, $\theta_{*}=k\left(\theta_{a}-\right.$ $\left.\theta_{g}\right) /\left[\ln \left(z / z_{0}\right)-\psi_{h}(z / L)\right]$, from the previous numerical time step. Note $\theta_{*}$ is negative for unstable conditions.

The functions for the stable regime 2 come from a slight modification of the linear relationship $\left[\phi_{m, h}=\right.$ $-5(z / L)]$ found in the Kansas program (Arya 1988) in order to ensure continuity with the functions of the more stable regime 1. A limit of -10 is used for both $\psi_{h}$ and $\psi_{m}$ in order to avoid the use of the Kansas-type functions for very stable conditions. The unstable functions of regime 4 are also from the Kansas field experiment (Paulson 1970). A lower limit of -10 is imposed to $z / L$ to prevent the use of these functions for very unstable conditions. 
The wind, temperature, and moisture are diagnosed at their typical observational height using the integrated dimensionless equations and assuming that $u_{*}, \theta_{*}$, and $q_{*}$ are constant with height. For instance, using Eq. (4) to obtain an expression for the wind at $z=10 \mathrm{~m}$ and dividing by the general form of the same Eq. (4) leads to

$$
u_{10 \mathrm{~m}}=u_{a} \frac{\ln \left(\frac{10}{z_{0}}\right)-\psi_{m}\left(\frac{10}{L}\right)}{\ln \left(\frac{z}{z_{0}}\right)-\psi_{m}\left(\frac{z}{L}\right)},
$$

where, as for the case of the transfer coefficients, the contribution of $\psi_{m}\left(z_{0} / L\right)$ has been neglected. An analogous derivation is used to diagnose the temperature and moisture at $2 \mathrm{~m}$ :

$$
\begin{aligned}
& \theta_{2 \mathrm{~m}}=\theta_{g}+\left(\theta_{a}-\theta_{g}\right) \frac{\ln \left(\frac{2}{z_{0}}\right)-\psi_{h}\left(\frac{2}{L}\right)}{\ln \left(\frac{z}{z_{0}}\right)-\psi_{h}\left(\frac{z}{L}\right)} \\
& q_{2 \mathrm{~m}}=q_{g}+\left(q_{a}-q_{g}\right) \frac{\ln \left(\frac{\rho c_{p} k u_{*} 2}{c_{s}}+\frac{2}{z_{l}}\right)-\psi_{h}\left(\frac{2}{L}\right)}{\ln \left(\frac{\rho c_{p} k u_{*} z}{c_{s}}+\frac{z}{z_{l}}\right)-\psi_{h}\left(\frac{z}{L}\right)} .
\end{aligned}
$$
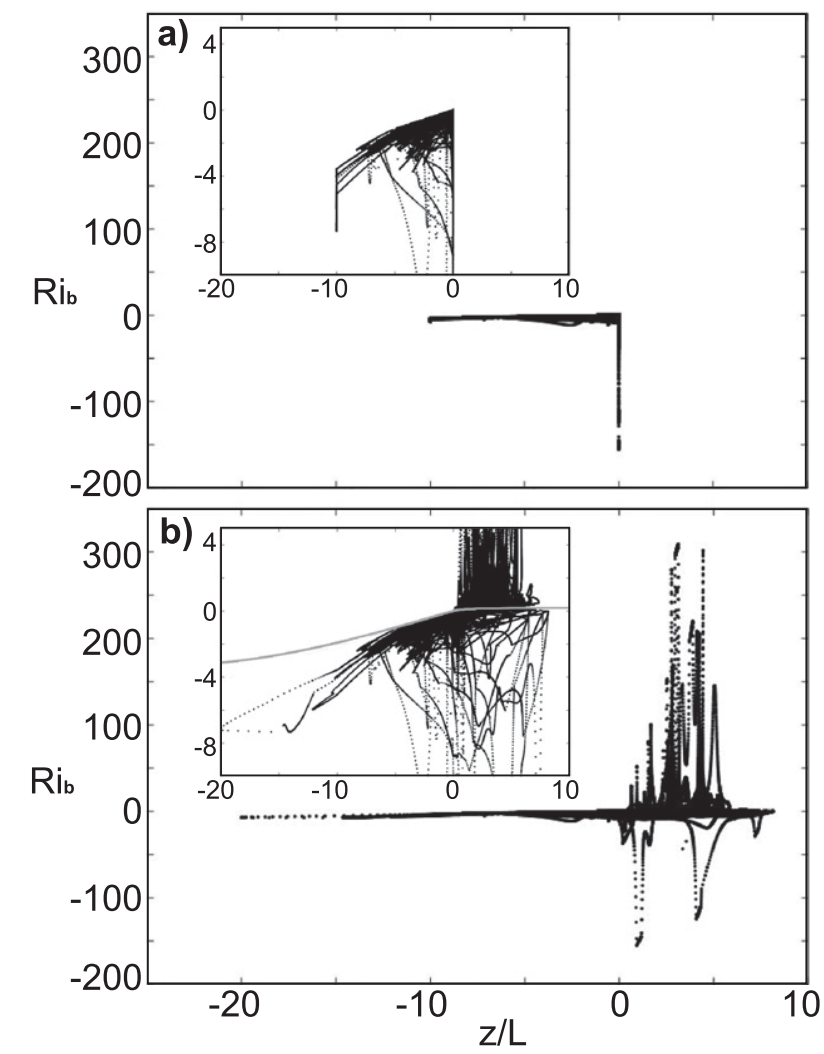

FIG. 1. (a) The $\mathrm{Ri}_{b}$ vs $z / L$ from the WRF output and (b) $\mathrm{Ri}_{b}$ vs $z / L$ diagnosed [Eq. (15)] with data from the WRF output. The gray line in (b) shows the theoretical relationship.

Additional restrictions to the allowable values of certain variables used to compute the fluxes and the near-surface variables are introduced in order to avoid undesired effects. For instance, $\ln \left(z / z_{0}\right)-\psi_{h}(z / L)$ is not allowed to be lower than 2 in order to avoid a high heat exchange coefficient, defined as the right-hand side of Eq. (2) except for the temperature differences, during unstable conditions in very thin surface layers with high roughness length. For similar reasons, $\psi_{h, m}$ is forced to be lower or equal to $0.9 \ln \left(z / z_{0}\right)$. In addition, the friction velocity is arithmetically averaged with its previous value in order to prevent large oscillations, and a lower limit of $u_{*}=0.1 \mathrm{~m} \mathrm{~s}^{-1}$ is imposed in order to prevent the heat flux from being zero under very stable conditions. It was considered that a smaller $u_{*}$ could potentially decouple the temperature of the atmosphere from the ground that starts cooling by radiation faster than observed in what is known as the runaway cooling effect (e.g., Louis 1979).

\section{b. Limitations of the present formulation}

Aside from the problems associated with the use of the Kansas similarity functions already mentioned, some other limitations in the above surface layer formulation can be pointed out. For instance, the lower limit $u_{*}=0.1 \mathrm{~m} \mathrm{~s}^{-1}$ used to avoid a potential runaway cooling effect, or the limits $\ln \left(z / z_{0}\right)-\psi_{h}(z / L)>2$ and $\psi_{m, h}(z / L) \leq 0.9 \ln \left(z / z_{0}\right)$ to avoid undesired effects in unstable conditions and very thin surface layers affect the self-consistency between the surface layer variables. For the case of $u_{*}$ the influence of the limit produces another negative impact, it prevents $u_{*}$ from reproducing the observed behavior since $u_{*}$ values below $0.1 \mathrm{~m} \mathrm{~s}^{-1}$ are common during the night (e.g., Shin and Hong 2011).

There are some other limitations that are not obvious at first glance, but are responsible for inconsistencies in the formulation. These limitations become evident in the dispersion diagram of $\mathrm{Ri}_{b}$ versus $z / L$ at one location wherein both variables were recorded every numerical time step (Fig. 1a). Only information regarding the unstable part is displayed since the formulation does not require computing $z / L$ in the two stable regimes (see previous section).

Theoretically, $\mathrm{Ri}_{b}$ and $z / L$ share the following relationship (e.g., Arya 1988):

$$
\mathrm{Ri}_{b}=\frac{z}{L} \frac{\ln \left(\frac{z}{z_{0}}\right)-\psi_{h}\left(\frac{z}{L}\right)}{\left[\ln \left(\frac{z}{z_{0}}\right)-\psi_{m}\left(\frac{z}{L}\right)\right]^{2}}
$$



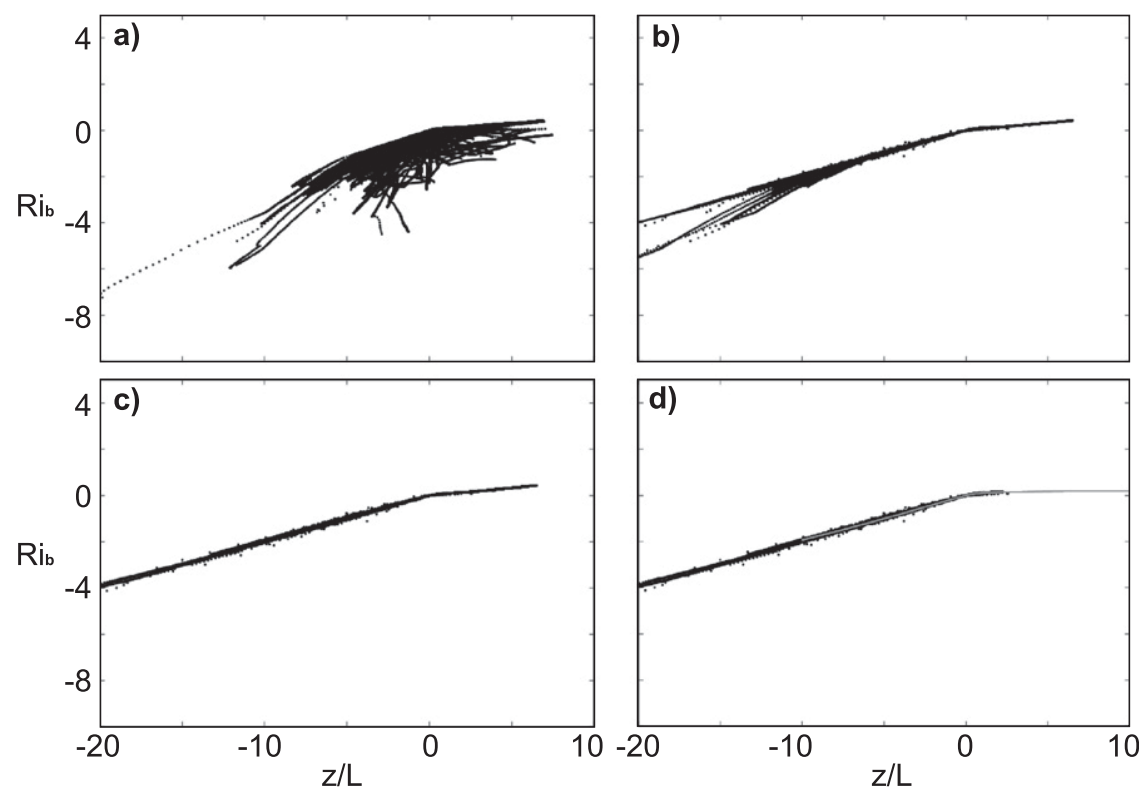

FIG. 2. The $\mathrm{Ri}_{b}$ vs $z / L$ after removing the effects of (a) $u_{*}=0.1 \mathrm{~m} \mathrm{~s}^{-1}$, (b) including the effects of moisture in the diagnosis of $z / L$, (c) removing the cases wherein $\ln \left(z / z_{0}\right)-\psi_{h}(z / L)=$ 2 , and (d) removing the instances with $\psi_{h, m}=-10$. The gray line in (d) shows the theoretical relationship.

which indicates that no scatter should be expected in the dispersion diagram of Fig. 1a. This is obviously not the case since noticeable dispersion can be appreciated (inset in Fig. 1a). The restrictions associated with the limit of $z / L=-10$ are clearly evident. A tendency to report zero $z / L$ values can also be appreciated. More information regarding the dispersion diagram between $\mathrm{Ri}_{b}$ and $z / L$, including the part associated with the stable regime, can be obtained using Eq. (15) to diagnose $z / L$ from the model output. The dispersion diagram between $\mathrm{Ri}_{b}$ and the diagnosed $z / L$ is shown in Fig. 1b. The tendency to report zero values of $z / L$ has disappeared but the scatter is even larger, especially for stable situations. There are also some situations wherein the surface layer is unstable according to $\mathrm{Ri}_{b}$ (negative values), but stable according to $z / L$ (positive values). In addition, there is a large discrepancy with the theoretical relationship between both variables (see inset of Fig. 1b).

Figure 2 provides a further understanding of the sources of the scatter. The dispersion diagram of $\mathrm{Ri}_{b}$ and the diagnosed $z / L$ after removing those instances wherein $u_{*}$ reaches its limit of $0.1 \mathrm{~m} \mathrm{~s}^{-1}$ is shown in Fig. 2a. A large part of the scatter has been eliminated. This indicates that the limit in $u_{*}$ was altering the values of $z / L$ through Eq. (15). However, inconsistencies in the stability definition are still evident since there are instances wherein both variables present different signs (Fig. 2a). The origin of this inconsistency is the use of the virtual potential temperature in the $\mathrm{Ri}_{b}$ calculation [Eq. (9)] and the potential temperature for $\theta_{*}$ in the $z / L$ calculation [Eq. (15)]. This is evident in Fig. 2b, which removes from the dispersion diagram those instances with $u_{*}=0.1 \mathrm{~m} \mathrm{~s}^{-1}$ and diagnoses $z / L$ with the virtual potential temperature. Inconsistencies in sign, and therefore stability, no longer appear. This change also reduces the scatter significantly. The inconsistencies in the stability definition are the reason for the large number of cases with $z / L=0$ during unstable conditions (Fig. 1a), since the surface layer scheme reports $z / L=0$ when $\mathrm{Ri}_{b}$ indicates a unstable surface layer (positive) and $z / L$ reports a stable surface layer (negative). Although a large part of the scatter has disappeared after the previous corrections, a noticeable dispersion in the unstable part is still evident (Fig. 2b). The reason for the largest dispersion that still remains is the $\operatorname{limit} \ln (z /$ $\left.z_{0}\right)-\psi_{h}(z / L)>2$ since the scatter is reduced after the situations exceeding the limit are removed (Fig. 2c). A better relationship between $\mathrm{Ri}_{b}$ and $z / L$ is obtained in stable conditions if those instances with $\psi=-10$ are also removed (Fig. 2d). This suppresses a change in the slope in the stable part and leads to a relationship that is in agreement with the theoretical behavior (gray line in Fig. 2d). Some scatter around the theoretical line is still evident, which is ultimately related to the use of information from the previous time step to calculate $z / L$ [Eq. (15)]. 


\section{c. Revised surface layer formulation}

The next two subsections describe the two sets of modifications introduced to 1 ) provide a better formulation of the similarity theory for strong unstable-stable conditions and 2) to mitigate the influence of the restrictions associated with limits.

\section{1) Similarity FUnCTIONS}

A first modification is introduced to provide a more suitable formulation of the similarity theory in stronger unstable/stable situations. For the unstable part, the $\psi$ functions proposed by F96 are used. The similarity functions present the asymptotic behavior predicted by the Monin-Obukhov theory in the convective limit, $|z / L| \rightarrow \infty$, and approaches to the Kansas functions' near-neutral conditions $|z / L| \rightarrow 0$. The following weighting function is used to calculate the integrated similarity functions:

$$
\psi_{h, m}=\frac{\psi_{\mathrm{Kh}, m}\left(\frac{z}{L}\right)+\left(\frac{z}{L}\right)^{2} \psi_{\mathrm{Ch}, m}\left(\frac{z}{L}\right)}{1+\left(\frac{z}{L}\right)^{2}}
$$

and $\psi_{\mathrm{Ch}, m}=3 / 2 \ln \left(y^{2}+y+1 / 3\right)-\sqrt{3} \arctan (2 y+1 / \sqrt{3})+$ $\pi / \sqrt{3}$ with $y=\left[1-\alpha_{h, m}(z / L)\right]^{1 / 3}$ and $\alpha_{m}=10$ and $\alpha_{h}=$ 34 (Grachev et al. 2000). Here, $\psi_{\mathrm{Kh}}$ represents the contribution of the Kansas-type functions (Paulson 1970) and $\psi_{\mathrm{Ch}}$ represents the convective contribution. See F96 for further details.

For the stable part, the formulation proposed by CB05 is adopted. The authors found an asymptotic behavior to a constant value in the similarity functions using data from the Cooperative Atmosphere-Surface Exchange Study-99 (CASES-99; Poulos et al. 2002). The $\psi$ functions proposed by $\mathrm{CB} 05$ are

$$
\begin{aligned}
& \psi_{m}=-a \ln \left\{\frac{z}{L}+\left[1+\left(\frac{z}{L}\right)^{b}\right]^{1 / b}\right\}, \\
& \psi_{h}=-c \ln \left\{\frac{z}{L}+\left[1+\left(\frac{z}{L}\right)^{d}\right]^{1 / d}\right\},
\end{aligned}
$$

with $a=6.1, b=2.5, c=5.3$, and $d=1.1$. These functions are valid for the entire range from neutral to very stable conditions. See CB05 for a more detailed explanation of the functions derivation.

The comparison of the old and new $\psi_{m}$ functions is shown in Fig. 3. The old formulation (black lines) reaches their limits $(z / L=-10$ for the unstable part and $\psi_{m}=-10$ for stable situations) for weak or moderately stabilities being constant for a large range (Figs. $3 a, b)$. On the contrary, the new formulation does not suffer from this limitation (thin gray lines). The unstable regime shows a more moderate increase (Fig. 3a) than the stable part (Fig. 3b). Both the unstable and stable functions show a similar evolution as the old formulation's near-neutral stabilities (Figs. 3c,d). The limit in the stable part is especially restrictive since it reaches the limit $\psi_{m}=-10$ around $z / L=2$ (Fig. $3 \mathrm{~d}$ ).

\section{2) LIMITS}

A slight modification in the definition of the bulk transfer coefficients [Eqs. (6), (7), and (8)] has been introduced:

$$
C_{d}=\frac{k^{2}}{\left[\ln \left(\frac{z+z_{0}}{z_{0}}\right)-\psi_{m}\left(\frac{z+z_{0}}{L}\right)+\psi_{m}\left(\frac{z_{0}}{L}\right)\right]^{2}},
$$

Where the extra terms $\psi_{m}\left(z_{0} / L\right), \psi_{h}\left(z_{0} / L\right)$ and $\psi_{m}\left(z_{l} / L\right)$ were dropped in Eqs. (6), (7), and (8) since for low values of $z / L$, and therefore $z_{0} / L$ or $z_{l} / L$, its contribution can be neglected. However, when high values of $z / L$ are allowed its contribution becomes more important due to the large slope of the integrated similarity functions near the origin (Figs. 3a,b). The effect that the extra term produces in the integrated similarity function for momentum is also shown in Fig. 3 (coarser gray lines). As expected, the contribution is small for weak stable/ unstable conditions (Figs. 3c,d), but becomes important for larger values of $|z / L|$ (Figs. 3a,b). In particular, it 

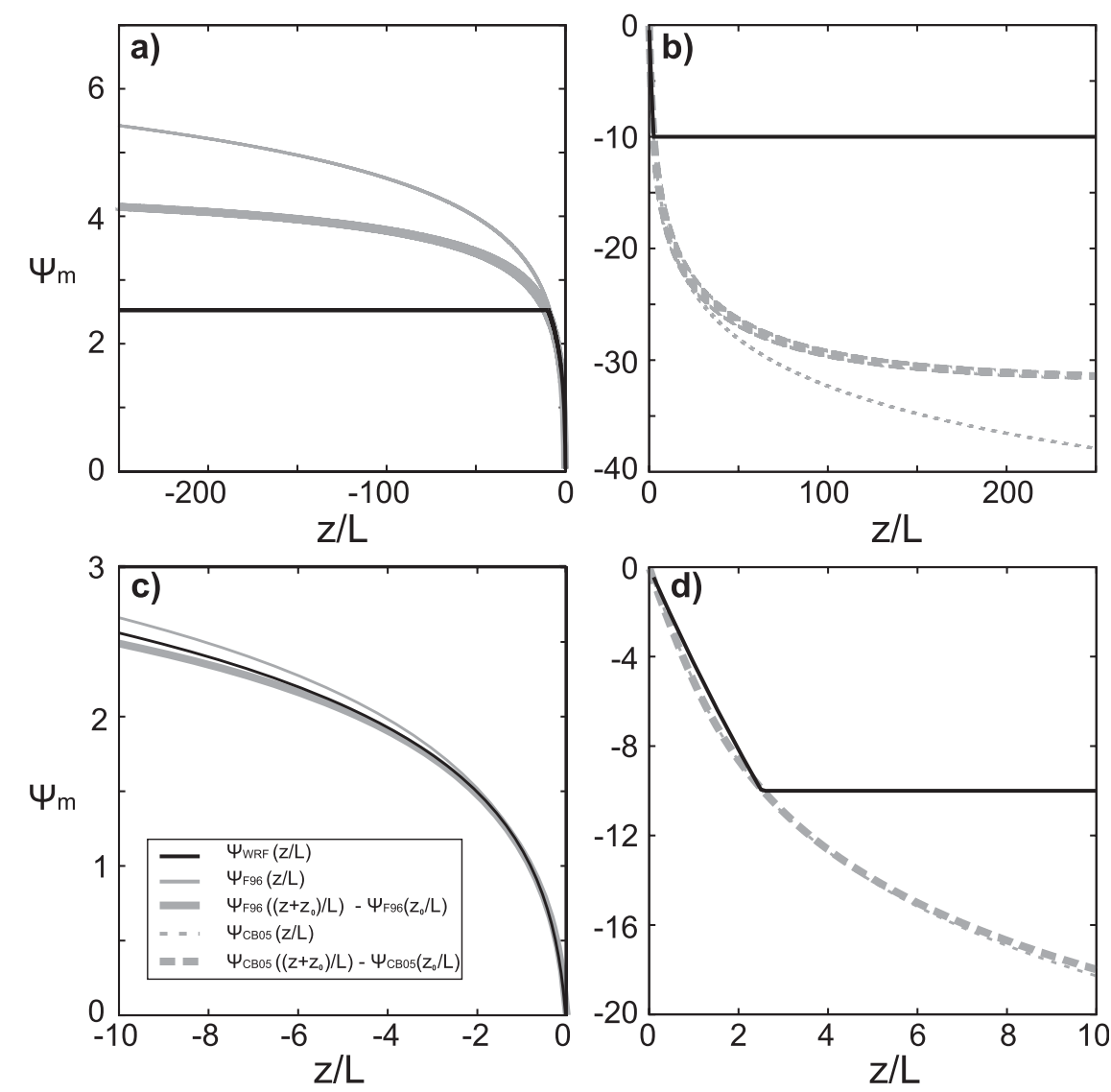

FIG. 3. Integrated similarity functions for momentum associated with (a) unstable and (b) stable conditions. (c),(d) The shape of the corrections near-neutral conditions. $\Psi_{\mathrm{WRF}}$ (black lines) is the integrated similarity function for momentum used in the old surface layer formulation, whereas $\psi$ F96 is the one used in the new formulation (thin gray lines). The integrated similarity functions including the extra term (thick gray lines) are calculated for $z=28 \mathrm{~m}$ and $z_{0}=0.15 \mathrm{~m}$.

produces a stronger asymptotic behavior in the integrated similarity functions.

The effects of the extra term shown in Fig. 3 have been calculated for a vertical level located at $z=28 \mathrm{~m}$ and for $z_{0}=0.15 \mathrm{~m}$. However, conclusions are valid for all the $z_{0}$ values allowable by WRF. Obviously, the larger $z_{0}$, the larger the differences between the old and the new formulation.

The extra terms prevent the surface layer formulation from having two undesired effects. The first one is related with the limit $\ln \left(z / z_{0}\right)-\psi_{h}(z / L) \geq 2$. We define

$$
\Psi_{T} \equiv \ln \left(\frac{z+z_{0}}{z_{0}}\right)-\psi_{h}\left(\frac{z+z_{0}}{L}\right)+\psi_{h}\left(\frac{z_{0}}{L}\right)
$$

The extra term not only prevents $\Psi_{T}$ from becoming too small but also from becoming negative, which would erroneously produce a different sign in $\mathrm{Ri}_{b}$ and $z / L$ since Eq. (16) should be substituted by

$$
\mathrm{Ri}_{b}=\frac{z}{L} \frac{\Psi_{T}}{\left[\ln \left(\frac{z+z_{0}}{z_{0}}\right)-\psi_{m}\left(\frac{z+z_{0}}{L}\right)+\psi_{m}\left(\frac{z_{0}}{L}\right)\right]^{2}}
$$

This can be appreciated in Fig. 4a. The integrated similarity functions without the extra term (dashed line) becomes negative around $z / L=-40$ whereas it is always positive with an asymptotic decrease to 0 if the extra term is included (gray line). The behavior of $\Psi_{T}$ as $|z / L|$ increases suggests that the limit of 2 can be avoided. On the basis of these considerations the limit in $\Psi_{T}$ has been removed in the new surface layer formulation. Again, results are shown for $z=28 \mathrm{~m}$ and $z_{0}=0.15 \mathrm{~m}$, but the conclusions are valid for all the allowable $z_{0}$ values.

The second undesired effect that is mitigated with the inclusion of the extra term in the integrated similarity function is associated with the limit $\psi_{h, m} \leq 0.9 \ln \left(z / z_{0}\right)$ or $\psi_{h, m}\left(z+z_{0} / L\right)-\psi_{h, m}\left(z_{0} / L\right) \leq \ln \left(z+z_{0} / z_{0}\right)$ in the 

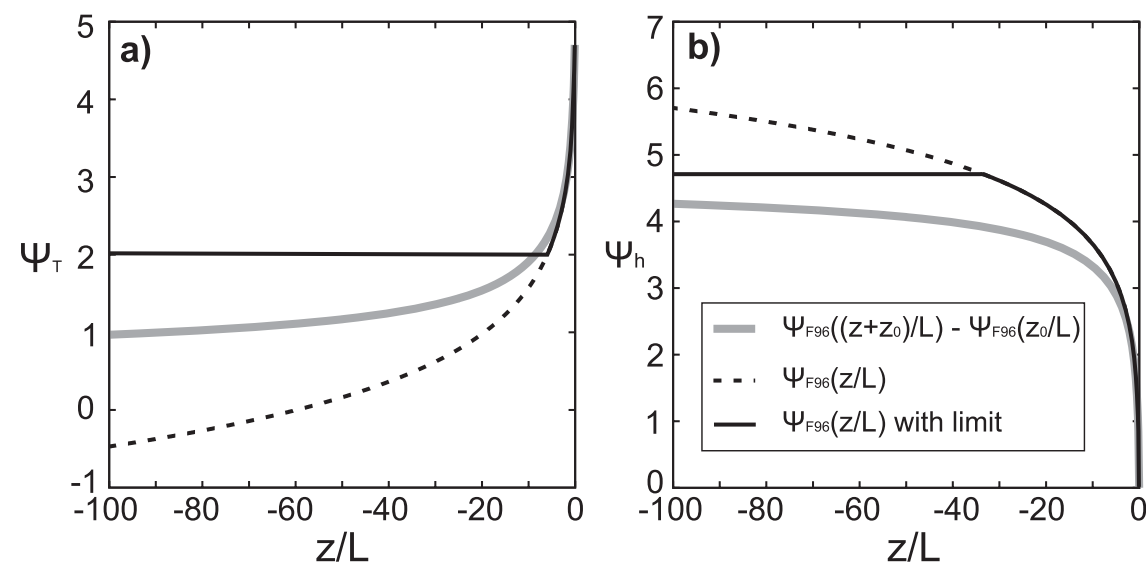

FIG. 4. (a) The $\Psi_{T}$ and (b) $\psi_{h}$ calculated with F96 integrated similarity functions (see legend). The effects of the extra term $\psi\left(z_{0} / L\right)$ are calculated using $z=28 \mathrm{~m}$ and $z_{0}=0.15 \mathrm{~m}$.

revised scheme. The slower increase of the new integrated similarity function makes it more difficult to reach this limit even though stronger unstable situations are allowed. Figure $4 \mathrm{~b}$ shows that the function would reach the limit at around $z / L=-35$, but the limit is not reached with the inclusion of the extra term. Actually, for a standard lower level at $28 \mathrm{~m}$ the limit is not reached by any of the allowable $z_{0}$ values.

It should be noticed that the upper limit of integration to obtain the integrated similarity functions, which was $z / L$ in the old formulation, is now changed to $\left(z+z_{0}\right) / L$. The reason for this change is to keep the thickness of the surface layer constant at $z$ in the calculation of the corrections. The other WRF surface layer scheme based on the similarity theory of the Eta Model (Skamarock et al. 2008) already has these limits of integration.

Another change is applied in order to obtain consistent values of $\mathrm{Ri}_{b}$ and $z / L$. This is achieved by solving Eq. (23) iteratively to obtain $z / L$ with an accuracy of 0.01 . Some authors use approximations to Eq. (23) in order to obtain $z / L$ as a function of $\mathrm{Ri}_{b}$ and therefore avoid the increase of computational time associated with an iterative procedure (e.g., Louis 1979). However, the most accurate relationships show errors of up to 14\% ( $\mathrm{Li}$ et al. 2010) and some tests have shown that the increase of the computational time due to the iterations is negligible.

The final change consists of reducing the $u_{*}$ limit to $0.001 \mathrm{~m} \mathrm{~s}^{-1}$. This change reduces the restrictions of the allowable values of $u_{*}$ by two orders of magnitude and is still expected to avoid the runaway cooling effect since the heat flux cannot be zero. The reduction of the limit also allows the formulation for reproducing typical nocturnal values of $u_{*}$, which can be below $0.1 \mathrm{~m} \mathrm{~s}^{-1}$ (e.g., Shin and Hong 2011).
The steps of the new formulation can be summarized as follows. First, $\mathrm{Ri}_{b}$ is calculated using Eq. (9). Then, a consistent value for $z / L$ is obtained by iterating Eq. (23). This value of $z / L$ defines the stability and is used to calculate the value of the $\psi_{h, m}$ functions [Eqs. (17), (18), and (19)] necessary to calculate the bulk transfer coefficients [Eqs. (20), (21), and (22)]. Finally, the values of the fluxes are computed [Eqs. (1), (2), and (3)] and the near-surface variables are diagnosed according to

$$
u_{10 \mathrm{~m}}=u_{a} \frac{\ln \left(\frac{10+z_{0}}{z_{0}}\right)-\psi_{m}\left(\frac{10+z_{0}}{L}\right)+\psi_{m}\left(\frac{z_{0}}{L}\right)}{\ln \left(\frac{z+z_{0}}{z_{0}}\right)-\psi_{m}\left(\frac{z+z_{0}}{L}\right)+\psi_{m}\left(\frac{z_{0}}{L}\right)},
$$

$$
\begin{aligned}
\theta_{2 \mathrm{~m}}= & \theta_{g}+\left(\theta_{a}-\theta_{g}\right) \\
& \times \frac{\ln \left(\frac{2+z_{0}}{z_{0}}\right)-\psi_{h}\left(\frac{2+z_{0}}{L}\right)+\psi_{h}\left(\frac{z_{0}}{L}\right)}{\ln \left(\frac{z+z_{0}}{z_{0}}\right)-\psi_{h}\left(\frac{z+z_{0}}{L}\right)+\psi_{h}\left(\frac{z_{0}}{L}\right)}, \\
q_{2 \mathrm{~m}}= & q_{g}+\left(q_{a}-q_{g}\right) \\
& \times \frac{\ln \left(\frac{\rho c_{p} k u_{*} 2}{c_{s}}+\frac{2}{z_{l}}\right)-\psi_{h}\left(\frac{2}{L}\right)+\psi_{h}\left(\frac{z_{l}}{L}\right)}{\ln \left(\frac{\rho c_{p} k u_{*} z}{c_{s}}+\frac{z}{z_{l}}\right)-\psi_{h}\left(\frac{z}{L}\right)+\psi_{h}\left(\frac{z_{l}}{L}\right)}
\end{aligned}
$$

\section{Numerical experiment}

The changes proposed in the surface layer formulation (section 2c) have been tested performing a series of 


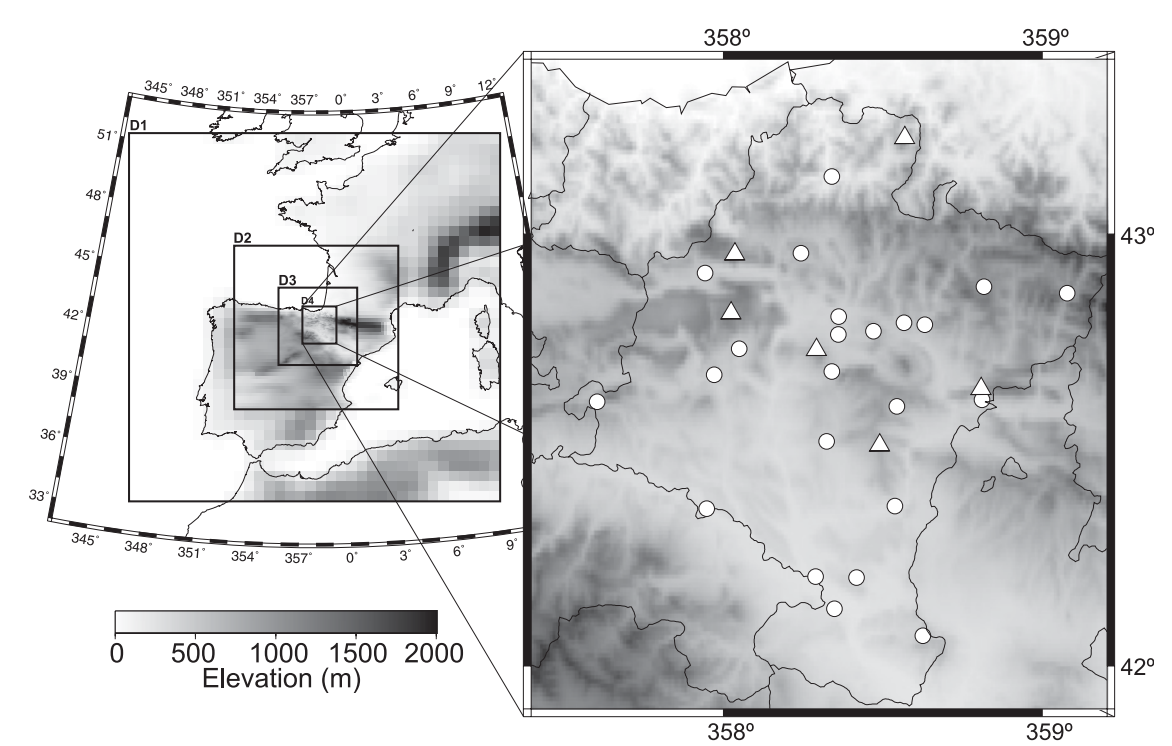

FIG. 5. Spatial configuration of the domains used in the WRF simulations. The topography of each domain is displayed at its particular horizontal resolution, 54 (D1), 18 (D2), 6 (D3), and $2 \mathrm{~km}$ (D4). The symbols in the zoomed-in area represent the location of the observational sites. Higher elevation sites are represented with a triangle.

numerical simulations with the version 3.1.1 of the WRF model (Skamarock et al. 2008). A total of four domains were nested using a two-way interaction to progressively reach a horizontal resolution of $2 \mathrm{~km}$ over a complex terrain region (Fig. 5) with a dense observational network (Jiménez et al. 2008, 2009a, 2010b). The high spatial resolution becomes necessary to accurately represent the complex terrain features of the region. A total of 29 observational sites recording standard near-surface variables were used for evaluation of the model performance (see zoomed-in area in Fig. 5).

The WRF model is initialized at the 0000 UTC of each day of the summer of 2002 (June-August, 92 days) and is run for $48 \mathrm{~h}$. Two sets of simulations are performed: one using the old surface layer formulation and the other with the revised scheme. The model output is stored every hour over the whole simulated domain that covers the whole area of study (zoomed-in area in Fig. 5). The large number of simulations allows us to average results in order to obtain a statistically robust characterization of the changes introduced with the new surface layer scheme. Additionally, a complete set of variables providing information on the surface layer is recorded every time step ( $\sim 11 \mathrm{~s})$ at the 29 observational sites. Saving the output every single time step allows us to obtain detailed information of the surface layer evolution.

The large-scale structure above the PBL is spectrally nudged (Miguez-Macho et al. 2004) in the coarser domain (D1, in Fig. 5) to the final National Centers for Environmental Prediction (NCEP) analysis used as initial and boundary conditions in the simulations. The PBL is parameterized with the Yonsei University scheme (Hong et al. 2006) with the first model level that defines the surface layer at $28 \mathrm{~m}(\eta=0.993)$. A simple soil scheme that diffuses the temperature in the ground has been used (Dudhia 1996; Dudhia et al. 2004). The scheme prescribes the soil moisture to a land-use-dependent value and thus allows us to more clearly isolate the effects introduced with the changes in the surface layer scheme. Further details on the physical and dynamical settings of the WRF simulation can be found in Jiménez et al. (2010a).

\section{Results}

The results are organized in three sections. The first one deals with the impacts in the simulation due to the reduction or suppression of limits and the changes introduced in the integrated similarity functions (section $4 a)$. The second one deals with the impact introduced in the fluxes and the near-surface variables (section $4 \mathrm{~b}$ ) and the last one with the impacts in the PBL dynamics and meteorological variables within this atmospheric layer (section 4c).

\section{a. Limits}

The influence of removing the limit in $\Psi_{T}$ is shown in Fig. 6 that displays the scatter diagram of the heat exchange coefficient against $\Psi_{T}$ for all the instances of the time series extracted at the 29 observational sites (Fig. 5). 


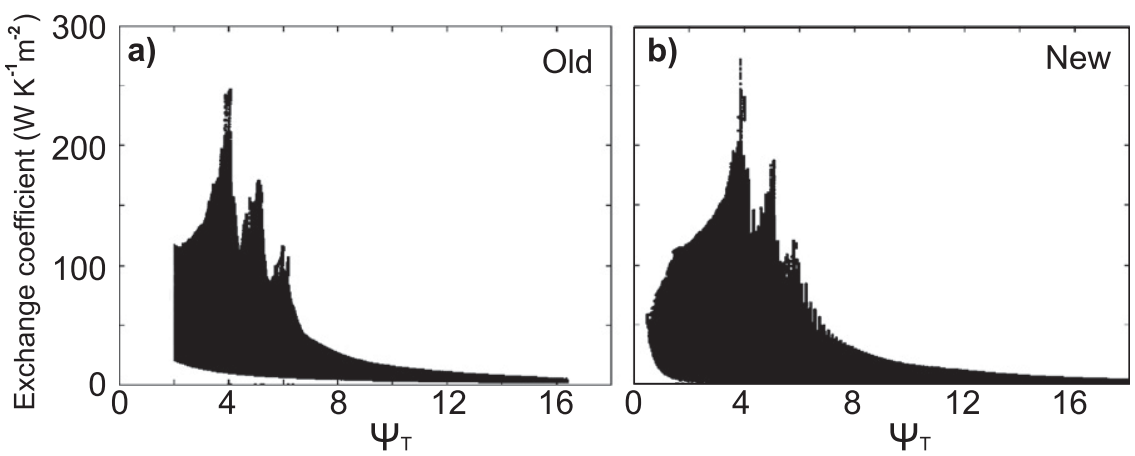

FIG. 6. Dispersion diagrams of the heat exchange coefficient vs $\Psi_{T}$ for (a) the old formulation and (b) the new formulation.

More than 41 millions pairs of $\Psi_{T}$ and heat exchange coefficient are shown (92 time series with 15552 instances at each one of the 29 observational sites). The old surface layer shows an abrupt behavior at lower values due to the lower limit of 2 (see section $2 a$ ). This does not happen in the new formulation, which shows a smoother distribution allowing for values below 2 without reaching the zero. This behavior could be expected on the basis of the considerations raised in the discussion of Fig. 4a.

The impact of allowing for very low values of $\Psi_{T}$ can be analyzed in the time series at a given observational site shown in Fig. 7. The time series are from the short WRF simulations initialized at 0000 UTC 19 July 2002. The new formulation shows nearly zero values of $\Psi_{T}$ around 1800 UTC of the first day of simulation (Fig. 7a).
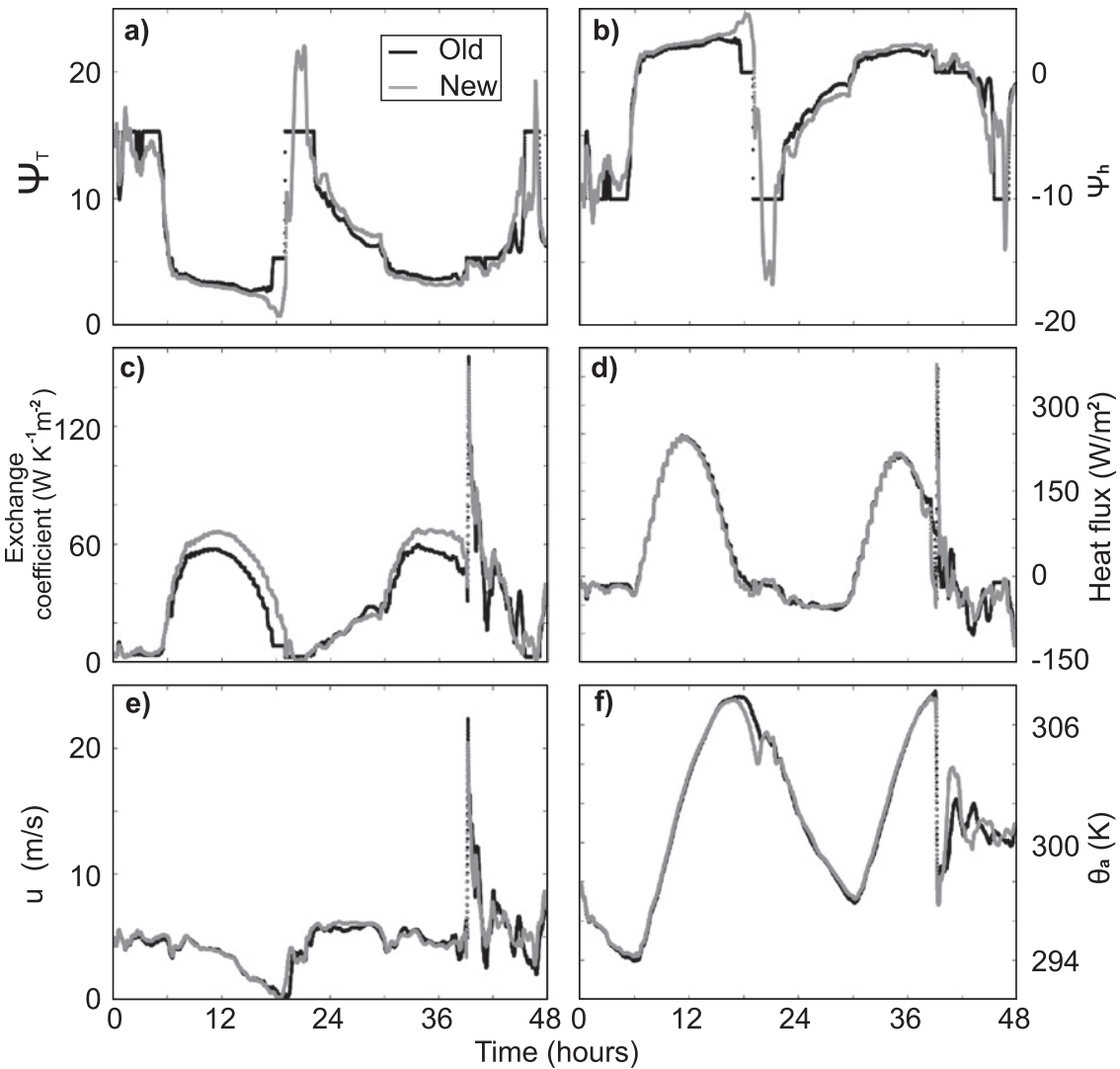

FIG. 7. Time series of (a) $\Psi_{T}$, (b) $\psi_{h}$, (c) heat exchange coefficient, (d) heat flux, (e) wind speed, and (f) potential temperature at one observational site from the short WRF simulations initialized at 0000 UTC 19 Jul 2002. 

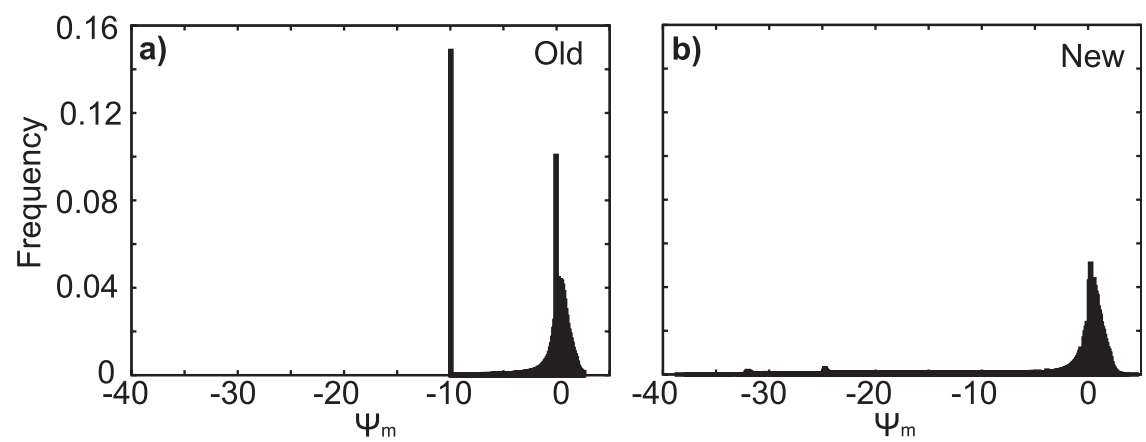

FIG. 8. Histograms of $\psi_{m}$ obtained with (a) the old formulation and (b) the new formulation.

On the contrary, the old formulation shows constant values of $\Psi_{T}$. Similar constant periods can be identified at the end of the afternoon of the second day of simulation (around $42 \mathrm{~h}$ ) and during the nights. These periods are associated with the limits of $\psi_{h}$ (Fig. 7b). One is associated with the lower limit of -10 and the other with 0 values of $\psi_{h}$ due to a different sign in $\mathrm{Ri}_{b}$ and $z / L$ related to the use of the virtual potential temperature in the former and the potential temperature in the later (see section $2 b)$. The periods wherein the atmosphere is neu$\operatorname{tral}(z / L=0)$ occur at the end of the afternoon revealing limitations in the old formulation to simulate the afternoon transition from unstable to stable conditions. The new formulation does not suffer from these limitations and shows an evolution of $\psi_{h}$ that is not influenced by constant values (Fig. 7b).

The motivation for applying a lower limit to $\Psi_{T}$ is to avoid a high heat exchange coefficient. However, this does not seem to be necessary in view of its time series (Fig. 7c). The coefficient does not show a high peak around 1800 UTC of the first day of simulation when $\Psi_{T}$ is very close to zero (Fig. 7a). The heat flux also shows a smooth evolution without any peaks at that time (Fig. 7d). Surprisingly, the fluxes are similar in both formulations in spite of the higher coefficient shown during the day by the revised scheme (Fig. 7c). This is related with a reduction of the temperature difference between the air and the ground associated with a reduction of the ground temperature and will be further discussed in the next section. The smooth behavior of the fluxes at 1800 UTC is a consequence of the low wind speeds simulated at that time (Fig. 7e), which are ultimately responsible for the decrease in $\Psi_{T}$ due to the increase of $\mathrm{Ri}_{b}$ [Eq. (9)]. Hence, although $C_{h}$ is large (low $\Psi_{T}$ ) this is compensated by the low value of $u$ [Eq. (2)] producing a smooth behavior of the heat flux (Fig. 7d).

All the analyzed cases of low $\Psi_{T}$ are associated with a low wind speed as in the previous case (Fig. 7) and, therefore, do not show a peak in the heat flux. The only peaks identified in the heat flux are associated with frontal passages. One of these cases occurs during the second day of simulation of the previous example (Fig. 7d). The high wind speed associated with the front (Fig. 7e) is responsible for the high peak in the flux (Fig. 7d). The drop of the temperature associated with the frontal passage is clearly visible in the time series of the surface layer potential temperature (Fig. 7f).

Periods with constant values of $\psi_{h, m}$ such as the ones shown in Fig. $7 \mathrm{~b}$ are found in most of the time series. Figure 8 shows the histogram of $\psi_{m}$ calculated with all the instances of the time series (again more than 41 million). The old formulation shows two peaks at 0 and -10 (Fig. 8a). The one at -10 shows a frequency of $15 \%$ indicating that a large percentage of the instances during stable conditions (i.e., nights) reach the limit in $\psi_{h, m}$. This is not surprising in view of the definition of $\psi_{h, m}$ for the stable regime $1, \mathrm{Ri}_{b} \geq 0.2$ (see section $2 \mathrm{a}$ ), which leads to $\psi_{h, m}$ lower than -10 if $z>e z_{0}$. The majority of the situations wherein the atmosphere is neutral $(z / L=0)$ occur before the transition to stable conditions at the end of the afternoon (some cases occur in the morning transition). The peaks cannot be identified in the $\psi_{m}$ distribution obtained with the new formulation that shows a smoother distribution around 0 and -10 (Fig. 8b).

Another restriction applied to the integrated similarity function is that $\psi_{h, m} \leq 0.9 \ln \left(z / z_{0}\right)$ or $\psi_{h, m}\left(z+z_{0} / L\right)-$ $\psi_{h, m}\left(z_{0} / L\right) \leq \ln \left(z+z_{0} / z_{0}\right)$ in the revised scheme. The analysis of the time series reveals that the limit is not reached either by the old formulation or by the revised one. This result shows the beneficial effects of the extra terms included in the integrated similarity functions (recall discussion of Fig. 4b), and suggests that a first level at $28 \mathrm{~m}(\eta=0.993)$ is adequate to prevent reaching this limit.

The last limit modified in the revised scheme affects the values of $u_{*}$. The old formulation presents a low limit of $0.1 \mathrm{~m} \mathrm{~s}^{-1}$, which has been replaced by $0.001 \mathrm{~m} \mathrm{~s}^{-1}$. The effects produced by this change are illustrated with the distribution of $u_{*}$ obtained with all the instances of 

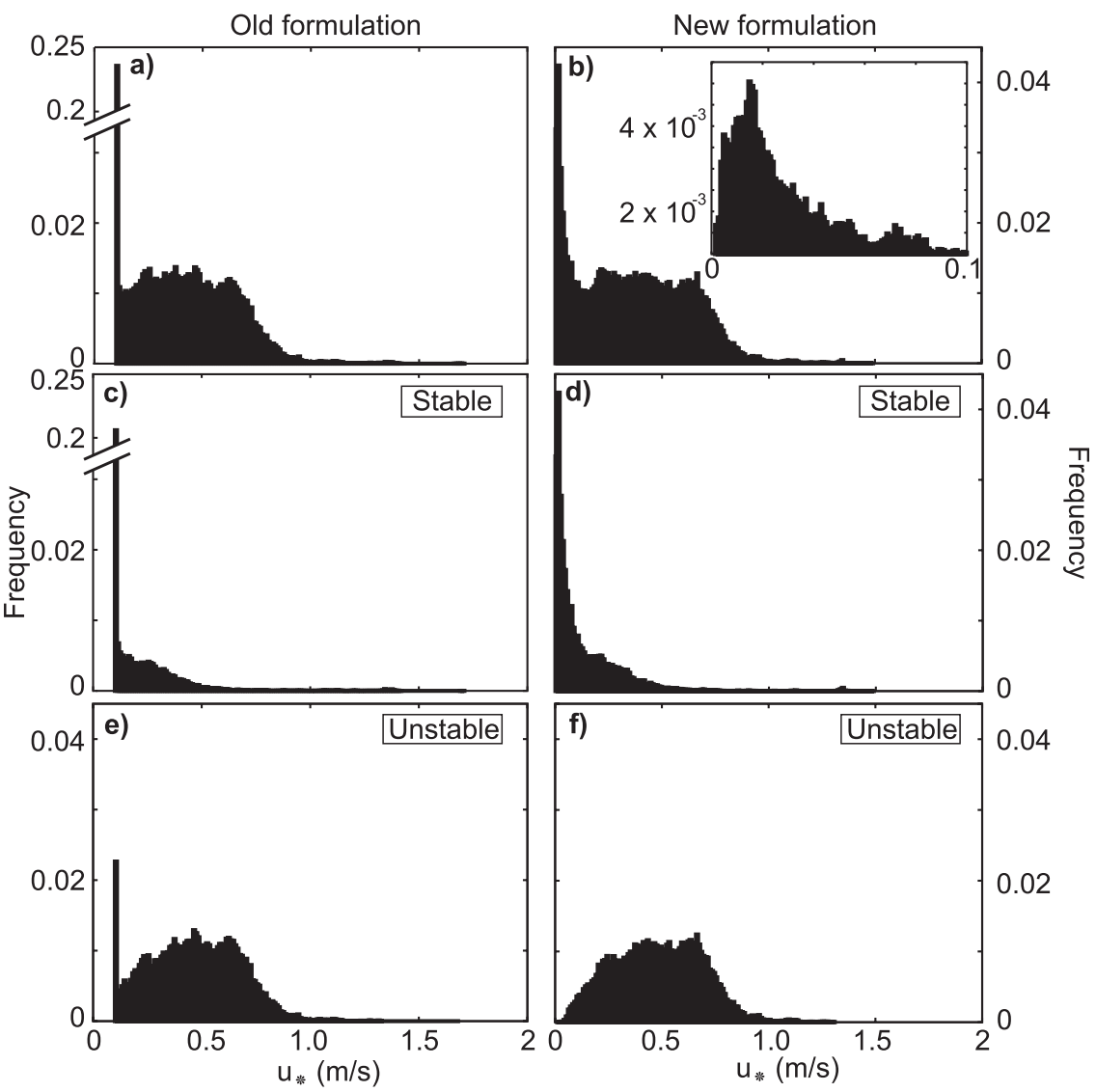

FIG. 9. Histograms of $u_{*}$ for (a) the old formulation and (b) the new formulation. The distribution calculated only for stable situations is shown for (c) the old formulation and (d) the new formulation. The distribution for unstable conditions under (e) the old formulation and (f) the new formulation. The inset in (b) shows the histogram for the lowest $u_{*}$ values at a discretization 10 times higher than the complete histogram. Note the break in the vertical scale in (a) and (c).

the 92 time series at one observational site (Fig. 9). The old formulation shows a peak at the limit around $25 \%$ of the time (Fig. 9a). The new formulation shows a smoother distribution at low $u_{*}$ (Fig. 9b). In particular, the distribution does not show a dominance of the values at the limit (see inset of in Fig. 9b). The histograms of $u_{*}$ calculated with the instances associated with a stable surface layer show that the old surface layer is dominated by the values at the limit (Fig. 9c) in contrast to the distribution provided by the new formulation (Fig. 9d). The unstable distribution is also affected by the low limit in $u_{*}$ (Fig. 9e) and, again, this does not happen with the revised scheme (Fig. 9f).

An interesting property that can be extracted from the histograms of $u_{*}$ associated with stable (Fig. 9d) and unstable (Fig. 9f) conditions is the presence of two friction velocity regimes with lower $u_{*}$ under stable situations and higher $u_{*}$ under unstable conditions. This different behavior is in part related to the contribution of the convective velocity (Beljaars 1995) that increases $u_{*}$ in the unstable regime. The two different regimes of $u_{*}$ suggest the presence of two different wind speed regimes since both variables share similar characteristics. These regimes are clearly recognized in the wind speed distribution of the observed and simulated wind speed (Fig. 10). Observations show a sharp distribution for the stable regime and a wider one for unstable conditions (Fig. 10a). Both simulations reproduce two wind regimes (Figs. 10b,c) but the stable distribution is wider than the observed one. The new formulation produces a narrower stable distribution with its maximum closer to the unstable counterpart more in agreement with observational evidence (Fig. 10a). However, it introduces a larger bias since the simulated wind was already too strong in the old formulation.

A potential limitation of reducing the limit of $u_{*}$ can be raised at this point. Low values of $u_{*}$ can reduce the turbulent heat flux decoupling the temperature of the ground from the air, and the ground starts cooling by 

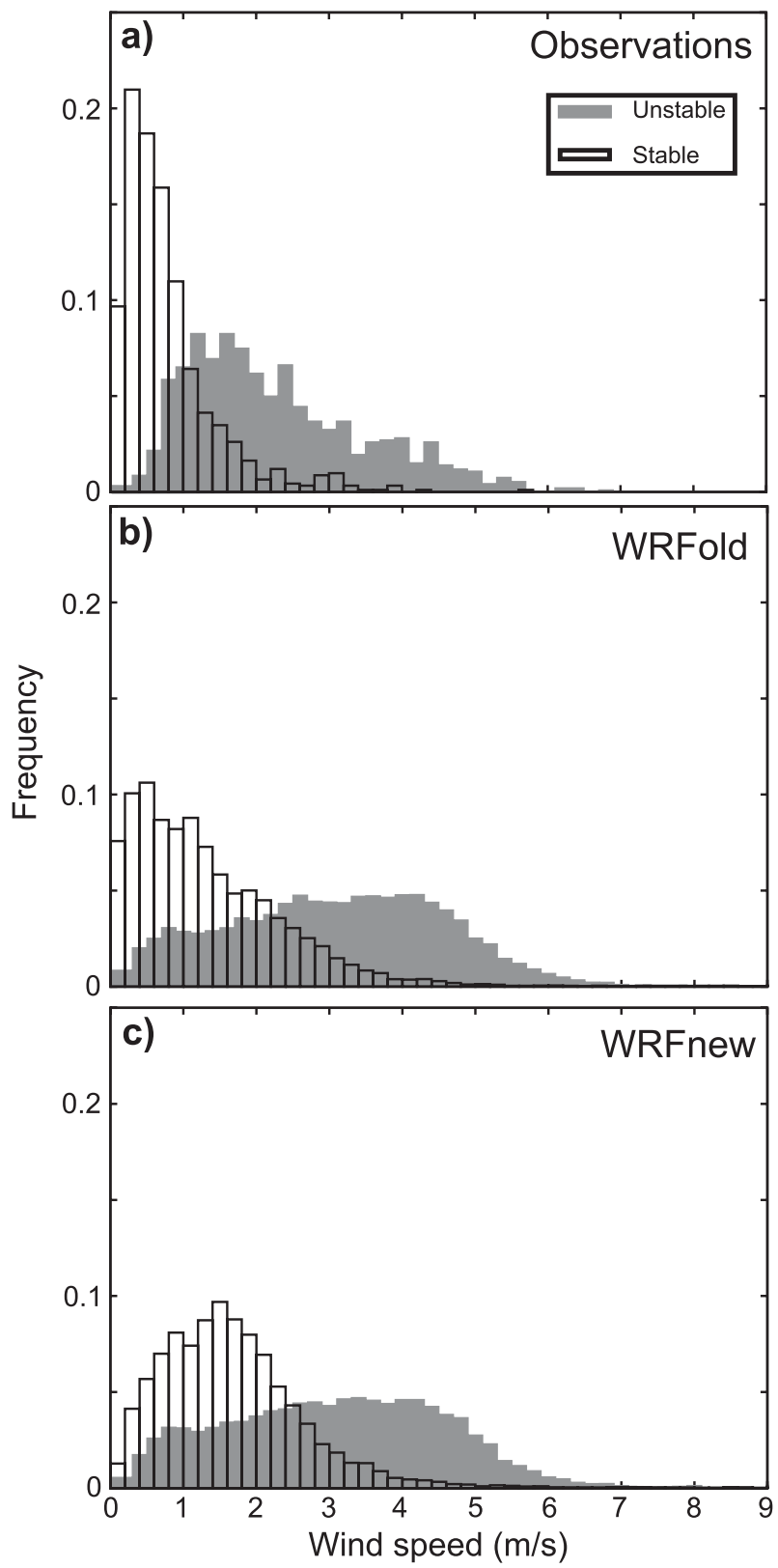

FIG. 10. Histograms of (a) observed wind speed as well as the simulated wind under (b) stable (line) and (c) unstable (shaded) conditions at the same location as Fig. 9.

radiation faster than observed (runaway cooling effect). However, this effect does not affect the simulation performed with the revised formulation. Figure 11 shows the histograms of the potential temperature difference between the air and the ground for all the instances of the time series wherein $u_{*}$ is lower or equal to $0.1 \mathrm{~m} \mathrm{~s}^{-1}$. Both histograms show a similar structure with a tendency in the one from the new formulation to increase the values of the temperature differences. This was expected since the new formulation is able to generate lower $u_{*}$ values. The important thing is that the new formulation does not produce inordinately large temperature differences, which would have been an indication of the runaway cooling effect.

\section{b. Fluxes and near-surface variables}

The diurnal evolution of the regional fluxes and diagnostic near-surface variables are shown in Fig. 12. The regional series have been obtained by averaging the simulated fields from the 92 WRF simulations. First, the simulated fields at each hour of the second day of simulation are averaged; then, the resulting mean fields at every hour are spatially averaged. The second day of simulation is used to calculate the diurnal evolution in order to avoid potential spinup problems at the beginning of the simulations as has been done in Jiménez et al. (2010a). Additionally, the averaged time series of the observed and simulated near-surface variables are shown in Fig. 13. Only information from the nearest grid point to the observational sites is used in this comparison. A more complete understanding of the changes introduced in the fluxes can be obtained by analyzing the diurnal evolution of the transfer coefficients $C_{d}, C_{h}$, and $C_{q}$. These have been calculated averaging the 92 time series at each one of the 29 observational sites and are displayed in Fig. 14. The sharper transition produced by the revised scheme at the end of the afternoon becomes evident in the three turbulent coefficients.

The new formulation produces a higher (lower) $u_{*}$ during the day (night) than the old formulation (Fig. 12a). The behavior of $u_{*}$ is therefore in agreement with the evolution of its associated bulk transfer coefficient $C_{d}$ (Fig. 14a). The revised scheme tends to increase (decrease) the surface wind speed during the night (day) since a decrease (increase) of $C_{d}$ produces a lower (higher) surface drag (Fig. 12b). The new formulation is in better agreement with the observed regional wind speed than the old formulation (Fig. 13a). The wind speed at the first model level shows similar changes in the diurnal evolution (not shown).

The prediction of a reduction of the wind speed amplitude (Fig. 12b) has been tested with observations from the meteorological network (Fig. 5). The difference between the maximum and minimum values is used to define the diurnal amplitude. The amplitude bias between the observed and simulated wind speed obtained with the old and the new formulation is shown in Figs. 15a and $15 \mathrm{~b}$, respectively. The sites located at mountain tops (triangles in Fig. 5) are excluded from the analysis of the diurnal wind amplitude since the simulation produces a diurnal wind speed cycle in opposite phase to the observed one at these locations (Jiménez et al. 2009b). The largest differences are found in the northern areas 

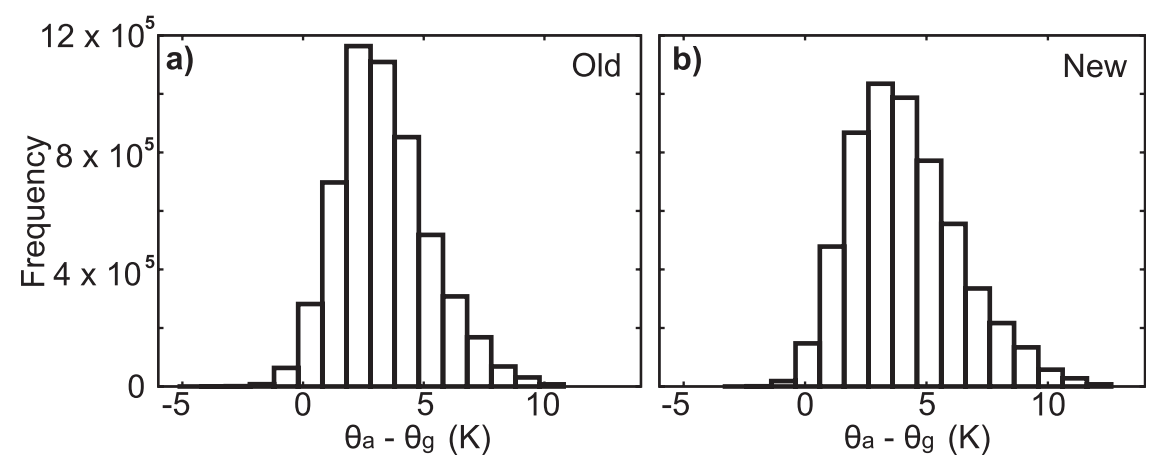

FIG. 11. Histogram of $\theta_{a}-\theta_{g}$ for the cases wherein $u_{*} \leq 0.1 \mathrm{~m} \mathrm{~s}^{-1}$ : (a) old and (b) new surface layer formulation.

because the simulated amplitude was too high (white circles in Fig. 15a) and this has been mitigated in the new formulation. Some portion of the error that is still present in the diurnal amplitude is related to wind speed biases. Both simulations show very similar wind biases since the new scheme tends to reduce the wind during the day and to increase it during the night leading to very similar mean (Fig. 12b). The one calculated with the new formulation is shown in Fig. 15c. The simulation tends to overestimate the wind speed over the valleys and to underestimate it at the mountain tops. This is related to the smoother topography used in the simulation (Jiménez 2009). The biases in the wind speed are introducing errors in the simulated amplitude since both the mean wind and the diurnal amplitude are correlated to a certain extent $(r=0.54)$. A quantification of the improvement obtained
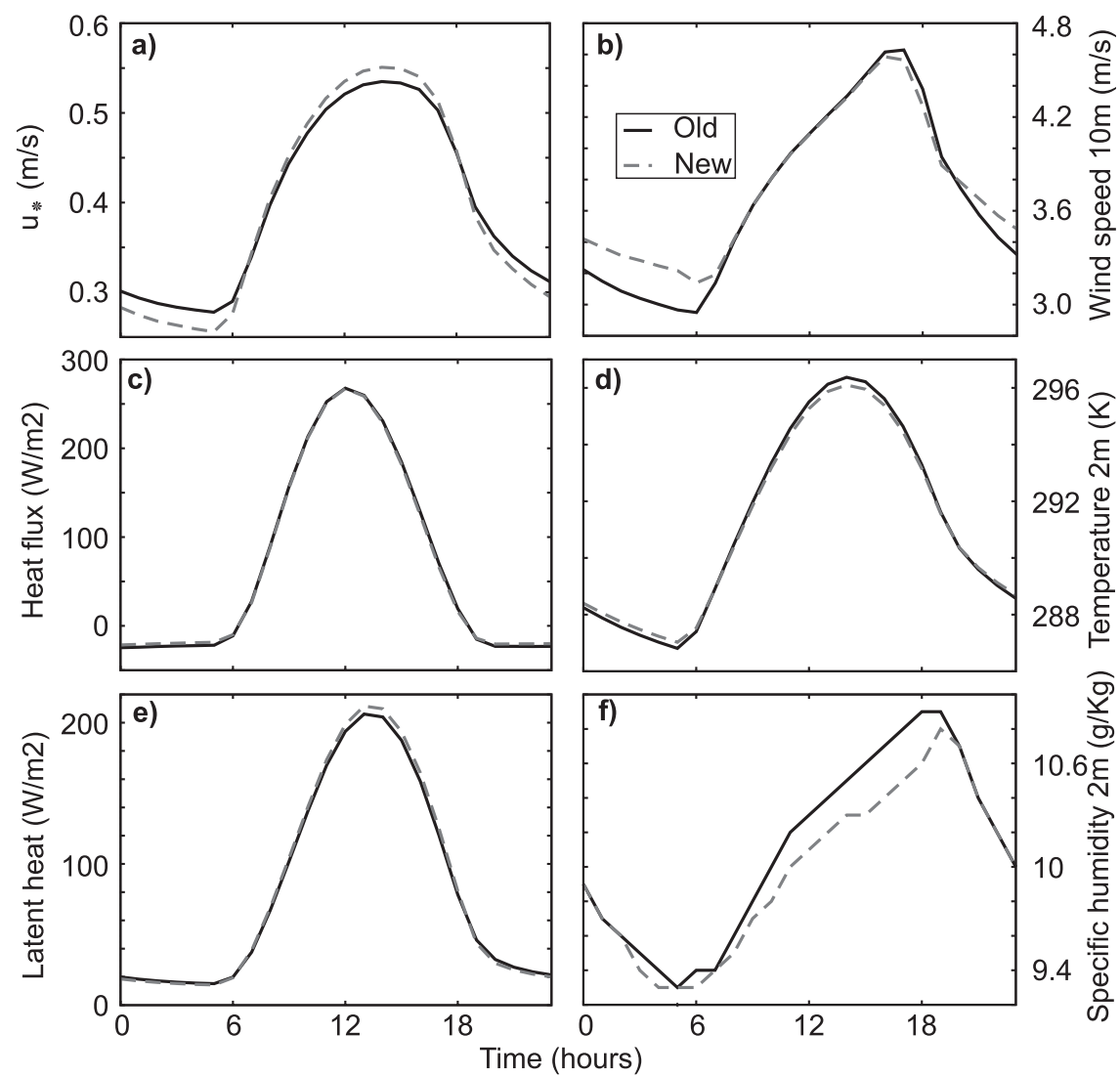

FIG. 12. Diurnal evolution of the regional time series for (a) $u_{*}$, (b) wind speed at $10 \mathrm{~m}$, (c) sensible heat flux, (d) temperature at $2 \mathrm{~m}$, (e) latent heat flux, and (f) specific humidity at $2 \mathrm{~m}$. 

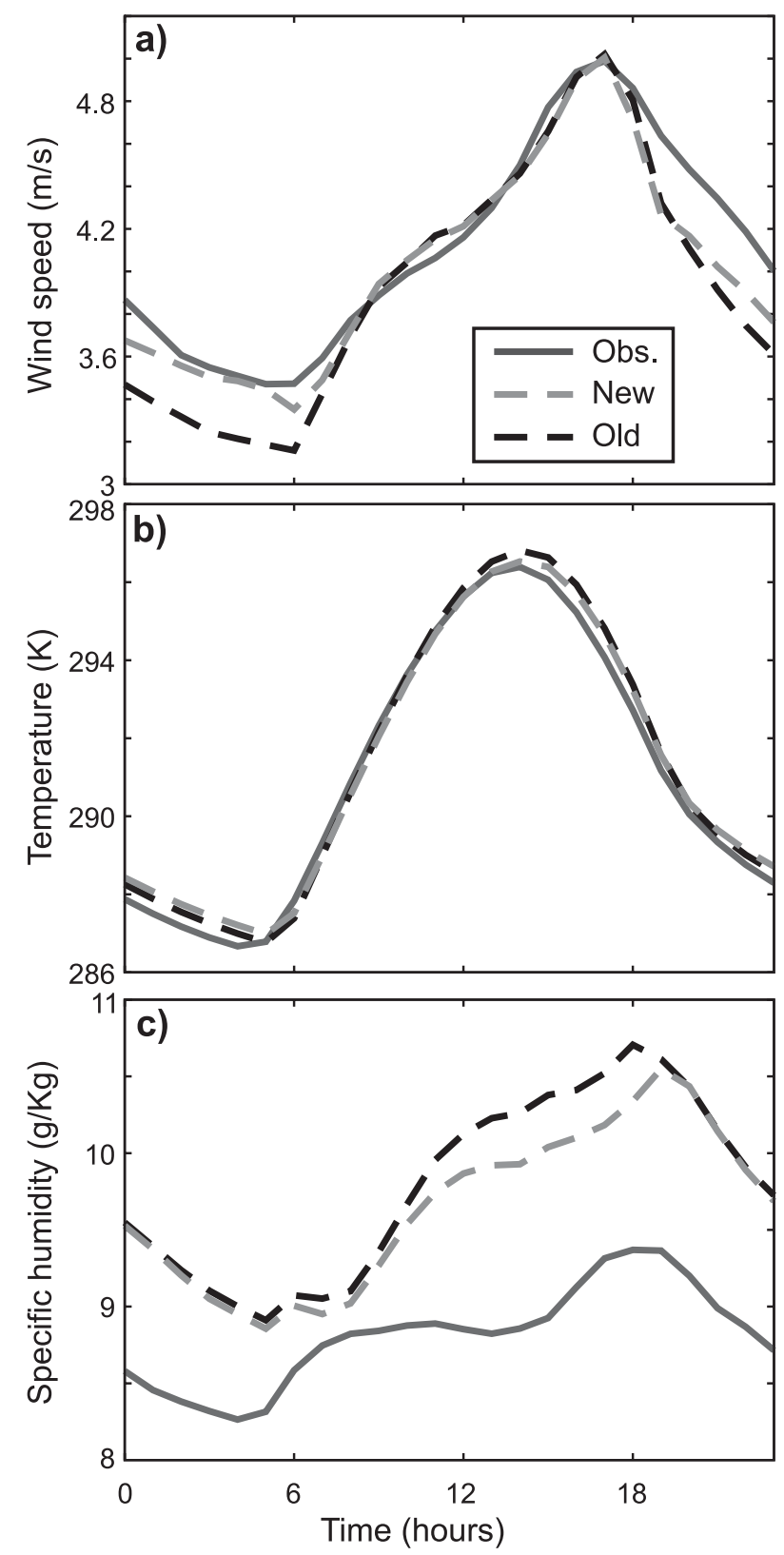

FIG. 13. Diurnal evolution of the averaged time series for (a) wind speed at $10 \mathrm{~m}$, (b) temperature at $2 \mathrm{~m}$, and (c) specific humidity at $2 \mathrm{~m}$ calculated with observations and the simulations (see legend). Information from the nearest grid point to the observational sites is used in the computation of the simulated time series. The specific humidity was available at three observational sites.

with the revised formulation has been obtained normalizing the difference between the absolute value of the wind speed amplitude biases by the observed wind speed amplitude at each site:

$$
A_{\text {improvement }}=\frac{\mid \text { Abias }_{\text {old }}|-| \text { Abias }_{\text {new }} \mid}{A_{\text {obs }}},
$$
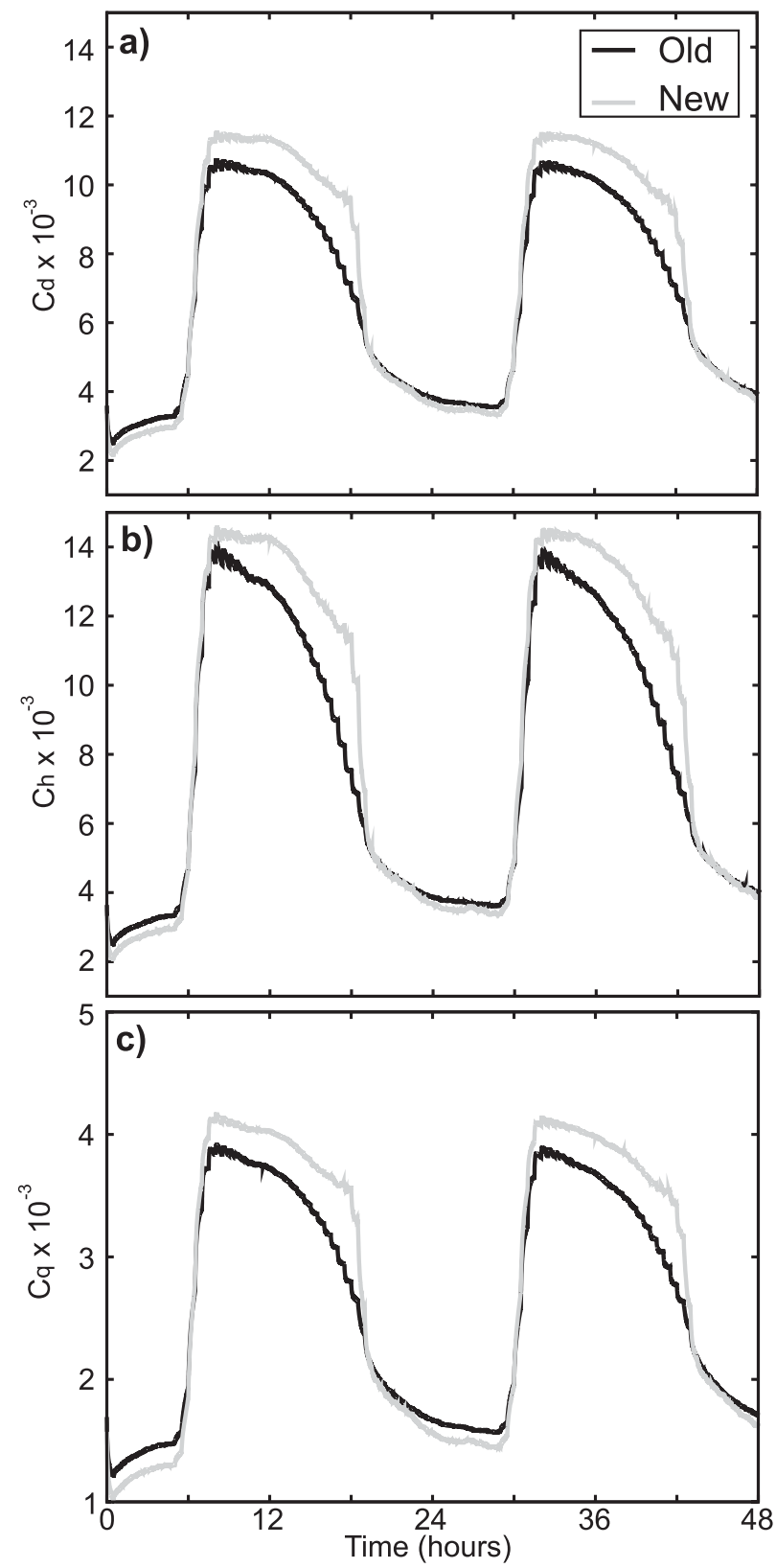

FIG. 14. (top to bottom) The $C_{d}, C_{h}$, and $C_{q}$ as a result of averaging the 2668 time series (92 simulations at each one of the 29 observational sites) for old and new surface layer formulation.. Notice the change of scale for $C_{q}$.

with $A$ standing for amplitude. Results are shown in Fig. $15 \mathrm{~d}$. There are a few sites (four) wherein the new surface layer formulation shows a worse performance (about $15 \%$, white diamonds in Fig. 15d); however, the revised scheme shows an overall better performance (black diamonds in Fig. 15d) with improvements of up to 50\%.

The heat flux does not show important variations in the new formulation (Fig. 12c). A slight tendency to 

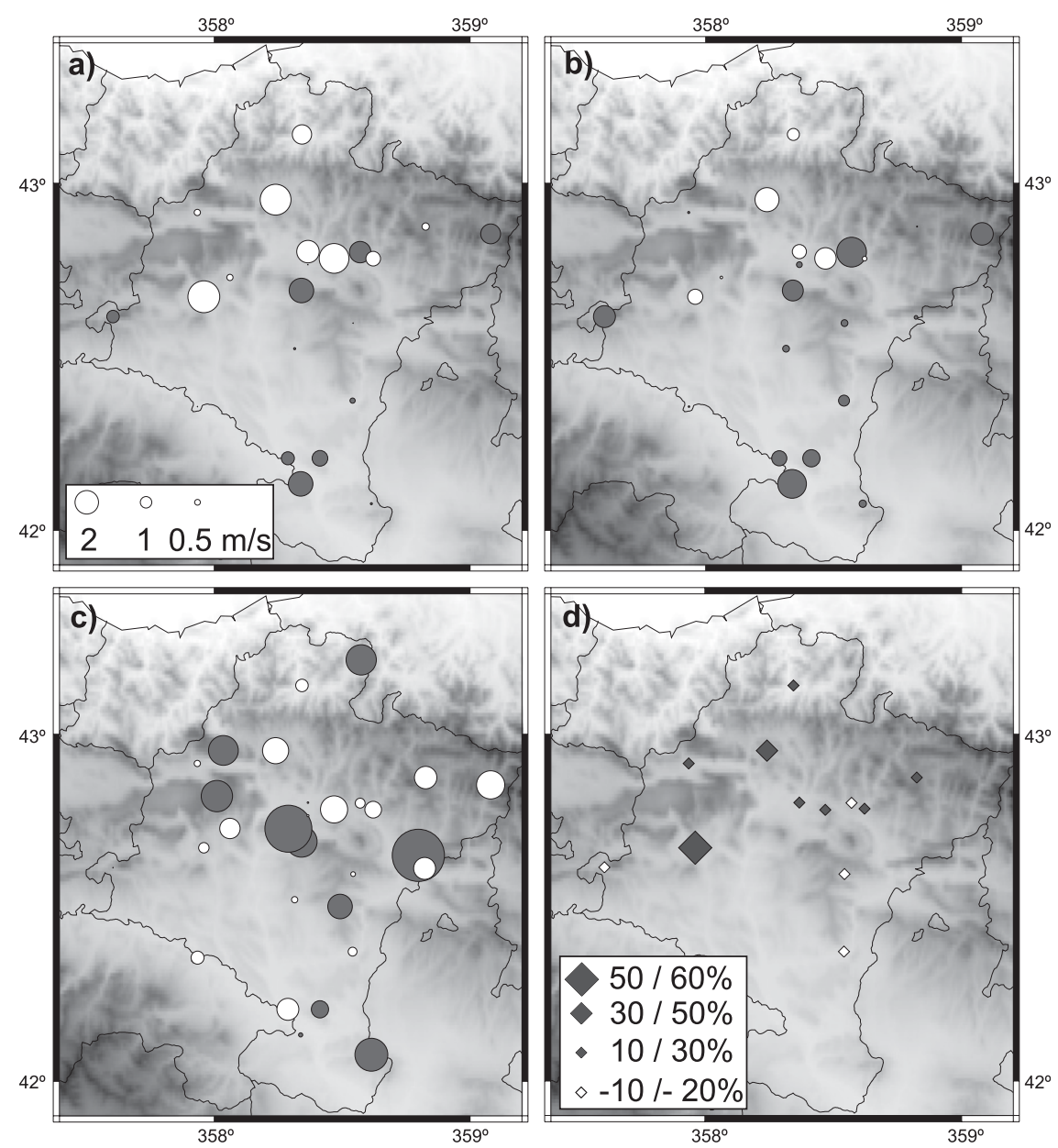

FIG. 15. Diurnal amplitude bias of the wind speed at $10 \mathrm{~m}$ calculated with the (a) old and (b) new surface layer formulation. (c) The wind speed bias, white (black) stands for wind speed overestimation (underestimation). (d) The difference of the absolute value of the normalized amplitude biases calculated using Eq. (27).

decrease during the night can be noticed in concordance with the lower values of $C_{h}$ (Fig. 14b), which overcomes the effects of an increase of the wind speed. The new scheme also tends to produce a reduction in the amplitude of the diurnal variations of the temperature at $2 \mathrm{~m}$ (Fig. 12d). Similar changes are evidenced using only information from the reduced number of observational sites (Fig. 13b). The effects are, however, smaller than for the wind speed. A quantification of the improvement of temperature at $2 \mathrm{~m}$ has been calculated in a similar way as with the wind speed, normalizing the absolute amplitude bias by the observed amplitude and subtracting the relative errors obtained with both surface layer schemes (Fig. 16). Again, the new scheme shows an overall better performance than the old one.

More important differences can be found in the latent heat flux (Fig. 12e). The new formulation tends to increase it during the day and to a lesser extent to reduce it during the night in agreement with its associated bulk transfer coefficient, $C_{q}$, evolution (Fig. 14c). The values of the specific humidity at $2 \mathrm{~m}$ show large modifications (Fig. 12f). The largest differences are found during the day wherein the new formulation reduces its values. A lower specific humidity at $2 \mathrm{~m}$ is in better agreement with the observations from the meteorological network (Fig. 13c), but there are still important biases perhaps associated with the use of a simple soil scheme in the WRF simulations.

A better understanding for the lower specific humidity at $2 \mathrm{~m}$ can be accomplished by analyzing the anomalies in the mean simulated fields at 1500 UTC (Fig. 17). The anomalies are calculated by subtracting the mean field from the old scheme from the mean field from the revised scheme. The reduction of the specific humidity occurs in most of the grid points of the domain (Fig. 17a) and it is related with a reduction of the ground temperature (Fig. 17b). A lower ground temperature leads 


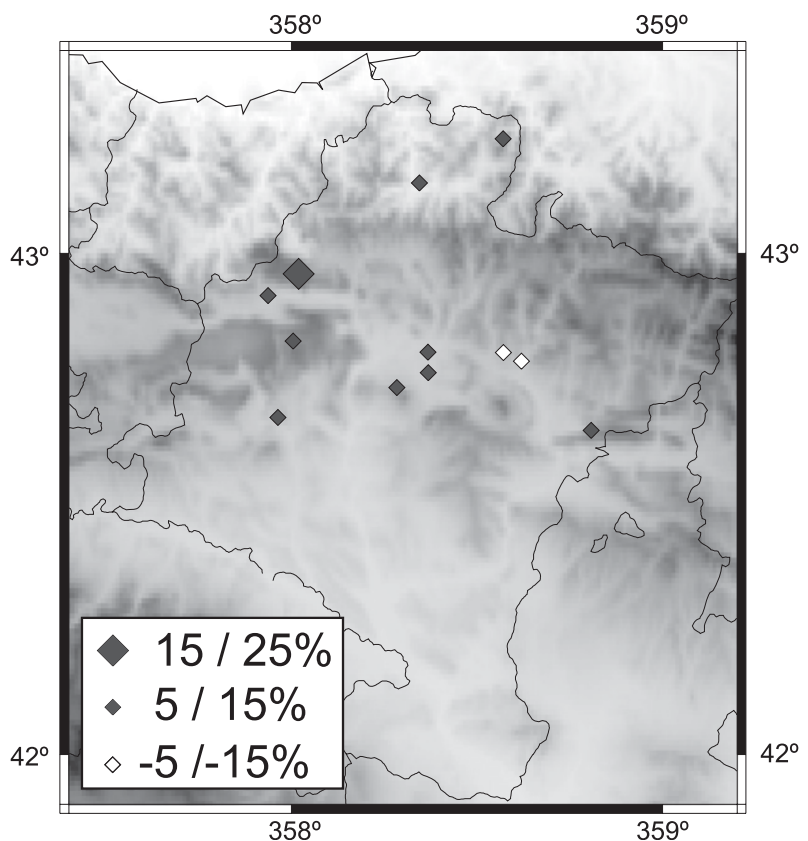

FIG. 16. As in Fig. 15d, but for the temperature at $2 \mathrm{~m}$.

to a lower saturated specific humidity at the ground $\left(q_{g}\right)$, and this reduces the interpolated values of the specific humidity at $2 \mathrm{~m}$ [Eq. (26)]. The lower ground temperature is associated with the higher efficiency of the fluxes during the day (Fig. 14), especially the one associated with moisture, which is responsible for an increase of the latent heat flux and, therefore, reduces the energy available for the ground flux.

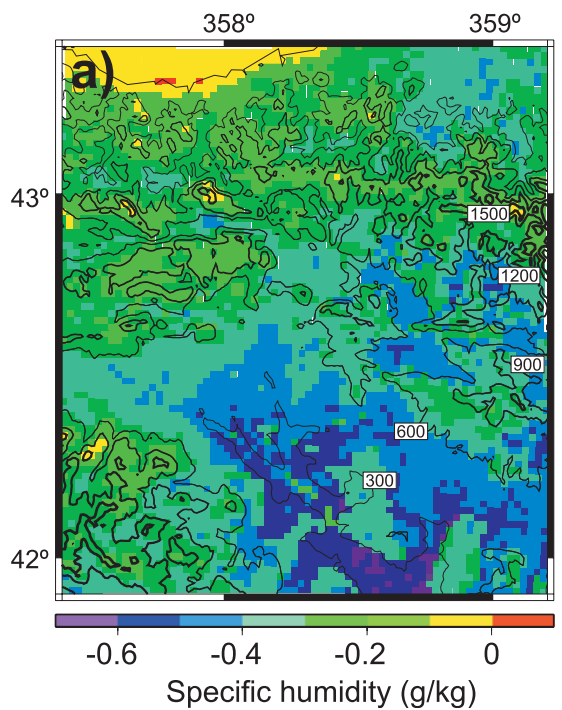

In concluding our analysis of the surface fluxes it is worth comparing results from both WRF simulations with the recent findings of Sorbjan (2010) and Sorbjan and Grachev (2010). It has been found that there exists an extremely stable regime for $\mathrm{Ri}>0.7$ wherein the turbulent fluxes should vanish. The revised scheme herein described leads to surface fluxes more in agreement with this finding than the old one. The mean heat flux calculated for those instances with $\mathrm{Ri}>0.7$ is $-12 \mathrm{~W} \mathrm{~m}^{-2}$ for the old scheme and $-1 \mathrm{~W} \mathrm{~m}^{-2}$ for the new one. The latent heat flux shows a similar reduction from 5 to $0.5 \mathrm{~W} \mathrm{~m}^{-2}$, and the averaged friction velocity of $0.1 \mathrm{~m} \mathrm{~s}^{-1}$ (the lower limit) is reduced to $0.01 \mathrm{~m} \mathrm{~s}^{-1}$ in the new scheme. The fluxes in the new formulation are therefore closer to vanishing than those from the old scheme. This is related to the influence of the limits. For $u_{*}$ the influence is very clear since the value in the old scheme is the value at the limit. The heat flux and the latent heat flux are closer to vanishing in the new scheme in part related to the smaller $u_{*}$, and in part to the smaller transfer coefficients of $C_{h}$ and $C_{q}$. The reduction is a consequence of the smaller values that the integrated similarity functions, $\psi_{m, h}$, can take in the new formulation as a result of removing the limit of -10 .

\section{c. PBL dynamics}

The impacts that the changes introduced in the surface layer formulation produce in the PBL dynamics are analyzed with the regional profiles of the wind speed, temperature, and specific humidity as well as the PBL height. The diurnal evolution of the profiles is calculated

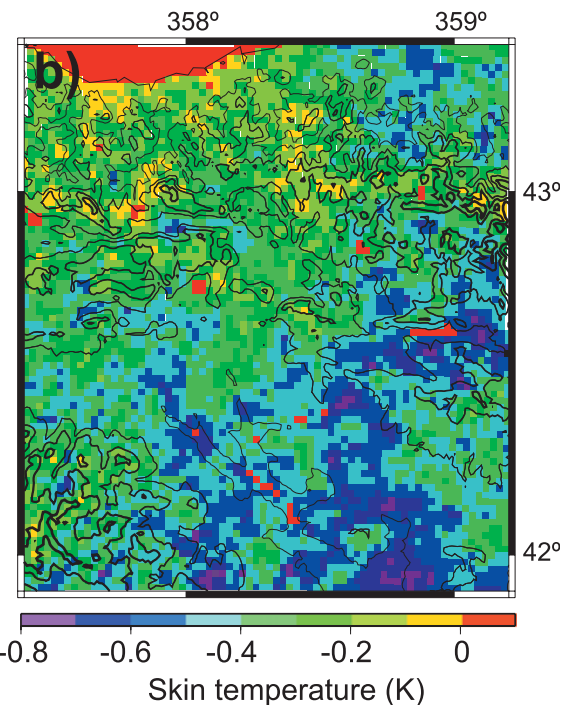

FIG. 17. Anomalies of the (a) specific humidity at $2 \mathrm{~m}$ and (b) skin temperature at 1500 UTC. The anomalies are calculated by subtracting the mean field at 1500 UTC obtained with the old formulation from the mean field at 1500 UTC from the new one. The topography is also shown (contour lines). 

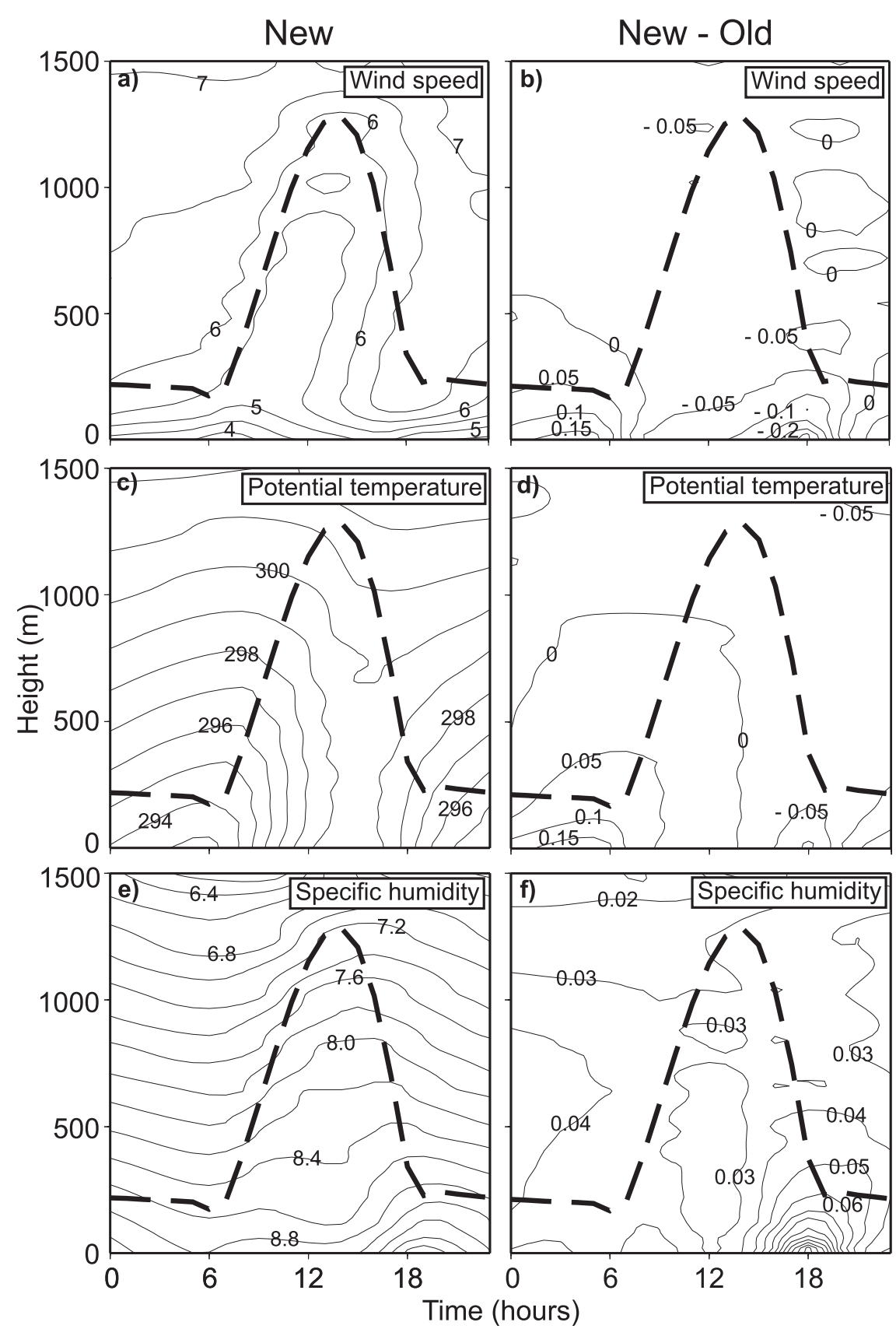

FIG. 18. Diurnal evolution of the (a) wind speed, (c) potential temperature, and (e) specific humidity calculated with the WRF simulation that uses the new surface layer scheme. (b),(d),(f) The differences between the new and the old formulation are also shown. Wind speed units are $\mathrm{m} \mathrm{s}^{-1}$, potential temperature $\mathrm{K}$, and specific humidity $\mathrm{g} \mathrm{kg}^{-1}$. The diurnal evolution of the PBL height is also shown (dashed line).

in a similar way as the regional fluxes and near-surface variables shown in Fig. 12. The diurnal evolution calculated with the simulation that uses the revised surface layer as well as the differences between both simulations are shown in Fig. 18. The wind speed and the potential temperature show similar characteristics (Figs. 18a,c).
The atmosphere shows a higher stratification during the night than during the day when the PBL is more homogeneous due to the higher mixing. The largest differences are found at lower levels during the afternoon transition, which is faster in the revised scheme, and during nighttime (Figs. 18b,c). The specific humidity shows a more 


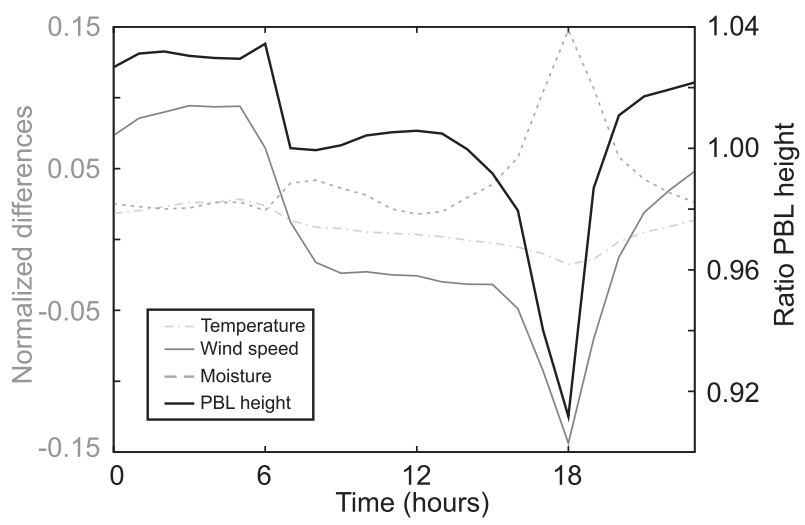

FIG. 19. Differences of the PBL-averaged potential temperature, wind speed, and specific humidity normalized by their particular diurnal amplitude. The ratio of the PBL heights is also shown. The differences are calculated subtracting the averaged values of the old formulation from those ones of the new formulation whereas the ratio is computed normalizing results from the new scheme by the values of the old scheme.

stratified atmosphere during the day (Fig. 18e) and the largest differences occur during the afternoon transition also near the surface (Fig. 18f).

The differences in the afternoon transition are ultimately related to the correction of the tendency in the old scheme to show a neutral atmosphere $(z / L=0)$ during the transition (e.g., Fig. 7b). This was associated with discrepancies in stability between $\mathrm{Ri}_{b}$ and $z / L$ as a result of the use of the virtual potential temperature in the former and the potential temperature in the later (see section 2). The correction of this erroneous treatment of the surface layer produces the largest differences in the bulk transfer coefficients at the end of the afternoon (Fig. 14), which are the main contributors for the differences in the PBL dynamics during the transition. On the other hand, the changes during the night are associated with the lower values that the friction velocity is allowed to reach in the new scheme and to the lower surface fluxes related to the suppression of the limits in the integrated similarity functions.

A quantification of the changes introduced with the new formulation in the PBL meteorological variables is summarized in Fig. 19. The differences of the PBLaveraged wind speed, potential temperature, and specific humidity calculated with the old scheme were subtracted from the averages of the new scheme and normalized by the mean diurnal amplitude. The ratio of the PBL heights from both simulations is also shown in Fig. 19. The PBL height is, on average, 9\% lower in the afternoon transition. This indicates that the PBL experiences a more abrupt transition in the new formulation. The differences can be up to $50 \%$ at some locations in southern areas of the region. The normalized wind speed shows a reduction of around $15 \%$ during the afternoon transition and a more moderate increase during the night (about $8 \%$ ). Some locations show a reduction of wind speed up to $62 \%$. Hence, the new formulation shows lower (higher) PBLaveraged wind than the old formulation during the day (night) in a similar evolution to the surface wind (Fig. 12b). The PBL-averaged specific humidity shows higher values in the revised scheme with a mean increase of about $15 \%$ during the afternoon transition (Fig. 19), but some locations present increases of up to $40 \%$. This is the opposite behavior of the near-surface specific moisture, which tends to decrease with the revised scheme (Fig. 12f). The apparent contradiction is related to the lower specific humidity of the ground simulated by the revised formulation (Fig. 17a) and, thus, the stronger moisture profile within the surface layer. The PBL-averaged temperature shows the smallest impact (Fig. 19). The largest differences occur during the night; the new formulation produces a slightly warmer PBL (about 3\%) in agreement with the higher surface temperature during the night (Fig. 12d).

\section{Conclusions}

A revised scheme for the WRF surface layer formulation has been presented. The new scheme provides a self-consistent formulation valid for the full range of atmospheric stabilities. The revised formulation produces a more abrupt afternoon transition than the old one. The old scheme tends to show a period with a neutral surface layer before the stable conditions are reached. The revised scheme does not suffer from this limitation and shows a sharper transition (Figs. 14 and 19), which is more in agreement with observational evidence and new parameterizations (e.g., Acevedo and Fitzjarrald 2001; Angevine and Mauritsen 2008).

The revised scheme also produces large impacts under stable conditions. The old formulation reaches the limits of $u_{*}$ and $\psi_{h, m}$ during a large percentage of the stable situations. This has been avoided in the revised formulation with an improved definition of the integrated similarity functions and a reduction of the lower $u_{*}$ limit. These changes provide surface fluxes more in agreement with the extremely stable regime recently suggested by Sorbjan (2010) and Sorbjan and Grachev (2010), and thus provide an improved land-atmosphere interaction under very stable situations.

The revised formulation leads to an overall improvement in the estimations of typical near-surface meteorological variables in terms of diurnal amplitude. This is especially the case of temperature at $2 \mathrm{~m}$. However, there are still important biases in the determination of these variables, such as the surface wind, which suggest that further improvements go beyond just the surface 
layer formulation. The surface wind biases over complex terrain are the subject of an ongoing study (Jiménez and Dudhia 2012). A more detailed comparison with observations including information on the surface fluxes would be desirable. The comparison should also test the performance of the revised surface layer formulation with a better land surface scheme (e.g., Chen and Dudhia 2001) where heat flux also follows Carlson and Boland (1978) like moisture here [Eqs. (7) and (8)]. Some preliminary tests in this direction suggest an even larger latent heat flux enhancement and a reduction of the sensible heat flux. Some sensitivity studies to the PBL parameterization should also be conducted to further explore the origins of the biases.

A sensitivity analysis to test the influence of the surface layer thickness, the first model layer, would provide further understanding of the surface layer formulation, which could lead to more improvements. Here, the lowest level is located a $z=28 \mathrm{~m}$, but it would be interesting to test the performance of the scheme using a lower level since this would reduce the uncertainties associated with the interpolation of the near-surface variables. Another potential improvement could be obtained by introducing a thermal roughness length, $z_{0 \mathrm{~h}}$, different than the one for momentum, $z_{0}$. This has already been incorporated in the Noah land surface scheme (Chen and Zhang 2009; Chen et al. 2010) and in updated versions of the WRF model. The impact that this change produces in the surface layer evolution and the PBL dynamics should be considered for future updates of the present formulation.

Acknowledgments. This investigation was partially supported by Projects CGL-2008-05093/CLI and CGL2011-29677-C02 and was accomplished within the Collaboration Agreement 09/490 between CIEMAT and NCAR as well as the Collaboration Agreement 09/153 between CIEMAT and UCM. NCAR is sponsored by the National Science Foundation. We thank the Navarra government for providing us with the observations used in this study. Discussion with Fei Chen and Peggy Lemone were helpful during this work. The authors would also like to thank Maria Tombrou for her comments regarding the similarity functions in unstable conditions. We would also like to thank the reviewers for their constructive comments.

\section{REFERENCES}

Acevedo, O. C., and D. R. Fitzjarrald, 2001: The early evening surface-layer transition: Temporal and spatial variability. J. Atmos. Sci., 58, 2650-2667.

Angevine, W. M., and T. Mauritsen, 2008: A new scheme for stable and fair-weather cumulus boundary layers in mesoscale models. Preprints, 2008 WRF Users' Workshop, Boulder, CO,
NCAR, 3-2. [Available online at www.mmm.ucar.edu/wrf/ users/workshops/WS2008/presentations/3-2.pdf.]

Arya, S. P., 1988: Introduction to Micrometeorology. Academic Press, $307 \mathrm{pp}$.

Beljaars, A. C. M., 1995: The parametrization of surface fluxes in large-scale models under free convection. Quart. J. Roy. Meteor. Soc., 121, 255-270.

- and A. A. M. Holtslag, 1991: Flux parametrization over land surfaces for atmospherics models. J. Appl. Meteor., 30, 327-341.

Brutsaert, W., 1992: Stability correction functions for the mean wind speed and temperature in the unstable surface layer. Geophys. Res. Lett., 19, 469-472.

Businger, J. A., J. C. Wyngaard, Y. Izumi, and E. F. Bradley, 1971: Flux-profile relationships in the atmospheric surface layer. J. Atmos. Sci., 28, 181-189.

Carlson, T. N., and F. E. Boland, 1978: Analysis of urban-rural canopy using a surface heat flux/temperature model. J. Appl. Meteor., 17, 998-1013.

Chen, F., and J. Dudhia, 2001: Coupling an advanced land surface hydrology model with the Penn State/NCAR MM5 modeling system. Part I: Model description and implementation. Mon. Wea. Rev., 129, 569-586.

and Y. Zhang, 2009: On the coupling strength between the land surface and the atmosphere: From viewpoint of surface exchange coefficients. Geophys. Res. Lett., 36, L10404, doi:10.1029/ 2009GL037980.

Chen, Y., K. Yang, D. Zhou, J. Qin, and X. Guo, 2010: Improving Noah land surface model in arid regions with an appropriate parameterization of the thermal roughness length. J. Hydrometeor., 11, 995-1006.

Cheng, Y., and W. Brutsaert, 2005: Flux-profile relationships for wind speed and temperature in the stable atmospheric boundary layer. Bound.-Layer Meteor., 114, 519-538.

Dudhia, J., 1996: A multilayer soil temperature model for MM5. Preprints, Sixth PSU/NCAR Mesoscale Model Users' Workshop, Boulder, CO, PSU/NCAR, 49-50.

—, D. Gill, K. Manning, W. Wang, and C. Bruyere, 2004: PSU/ NCAR mesoscale modeling system tutorial class notes and user's guide: MM5 modelling system version 3. PSU/NCAR, $390 \mathrm{pp}$.

Dyer, A. J., 1967: The turbulent transport of heat and water vapor in unstable atmosphere. Quart. J. Roy. Meteor. Soc., 93, 501-508. 1974: A review of flux-profile relationships. Bound.-Layer Meteor., 7, 363-372.

— gradient relationships at the 1976 ITCE. Bound.-Layer Meteor., 22, 3-19.

Fairall, C. W., E. F. Bradley, D. P. Rogers, J. B. Edson, and G. S. Young, 1996: Bulk parameterization of air-sea fluxes for Tropical Ocean Global Atmosphere Coupled-Ocean Atmosphere Response Experiment. J. Geophys. Res., 101 (C2), 3747-3764.

,-- J. E. Hare, A. A. Grachev, and J. B. Edson, 2003: Bulk parametrization of air-sea fluxes: Updates and verification for the COARE algorithm. J. Climate, 16, 571-591.

García-Bustamante, E., J. F. González-Rouco, J. Navarro, E. Xoplaki, P. A. Jiménez, and J. P. Montávez, 2011: North Atlantic atmospheric circulation and surface wind in the Northeast of the Iberian Peninsula: Uncertainty and long term downscaled variability. Climate Dyn., in press.

Grachev, A. A., C. W. Fairall, and E. F. Bradley, 2000: Convective profile constants revisited. Bound.-Layer Meteor., 94, 495-515.

Grell, G. A., J. Dudhia, and D. R. Stauffer, 1994: A description of the fifth-generation Penn State/NCAR Mesoscale Model (MM5). NCAR Tech. Note NCAR TN-398-1-STR, 117 pp. 
Hicks, B. B., 1976: Wind profile relationships from the Wangara experiments. Quart. J. Roy. Meteor. Soc., 102, 535-551.

Holtslag, A. A. M., and H. A. de Bruin, 1988: Applied modelling of the nighttime surface energy balance over land. J. Appl. Meteor., 27, 689-704.

Hong, S.-Y., Y. Noh, and J. Dudhia, 2006: A new vertical diffusion package with an explicit treatment of entrainment processes. Mon. Wea. Rev., 134, 2318-2341.

Izumi, Y., 1971: Kansas 1968 field program data report. Tech. Rep. Environmental Research Paper 379, Air Force Cambridge Research Laboratories, Bedford, MA, 86 pp.

Jiménez, P. A., 2009: Analysis of surface wind over complex terrain: A dynamical downscaling study with the WRF model. Ph.D. thesis, Universidad Complutense de Madrid, 199 pp.

— solved and unresolved topographic effects on surface wind in the WRF model. J. Appl. Meteor. Climatol., 51, 300-316.

— , J. F. González-Rouco, J. P. Montávez, J. Navarro, E. GarcíaBustamante, and F. Valero, 2008: Surface wind regionalization in complex terrain. J. Appl. Meteor. Climatol., 47, 308-325.

- — — — - E. García-Bustamante, and J. Navarro, 2009a: Climatology of wind patterns in the northeast of the Iberian Peninsula. Int. J. Climatol., 29, 501-525.

—, J. P. Montávez, E. García-Bustamante, J. Navarro, J. M. Jiménez-Gutiérrez, E. E. Lucio-Eceiza, and J. F. GonzálezRouco, 2009b: Diurnal surface wind variations over complex terrain. Fís. Tierra, 21, 79-91.

—, J. F. González-Rouco, E. García-Bustamante, J. Navarro, J. P. Montávez, J. Vilà-Guerau de Arellano, J. Dudhia, and A. Roldán, 2010a: Surface wind regionalization over complex terrain: Evaluation and analysis of a high-resolution WRF numerical simulation. J. Appl. Meteor. Climatol., 49, 268-287.

, — - J. Navarro, J. P. Montávez, and E. García-Bustamante, 2010b: Quality assurance of surface wind observations from automated weather stations. J. Atmos. Oceanic Technol., 27, $1101-1122$.

Jingyong, Z., W.-C. Wang, and L. R. Leung, 2008: Contribution of land-atmosphere coupling to summer climate variability over the contiguous United States. J. Geophys. Res., 113, D22109, doi:10.1029/2008JD010136.

Li, Y., Z. Gao, D. H. Lenschow, and F. Chen, 2010: An improved approach for parameterizing turbulent transfer coefficients in numerical models. Bound.-Layer Meteor., 137, 153-165.

Louis, J. F., 1979: A parametric model of vertical eddy fluxes in the atmosphere. Bound.-Layer Meteor., 17, 187-202.

Mahrt, L., and J. Sun, 1995: The subgrid velocity scale in the bulk aerodynamic relationship for spatially averaged scalar fluxes. Mon. Wea. Rev., 123, 3032-3041.

Miguez-Macho, G., G. L. Stenchikov, and A. Robock, 2004: Spectral nudging to eliminate the effects of domain position and geometry in regional climate model simulations. J. Geophys. Res., 109, D13104, doi:10.1029/2003JD004495.

Monin, A. S., and A. M. Obukhov, 1954: Basic laws of turulent mixing in the atmosphere near the ground. Tr. Inst. Teor. Geofiz. Akad. Nauk SSSR, 24, 1963-1987.

Obukhov, A. M., 1946: Turbulence in thermally non-homogeneous atmosphere. Tr. Inst. Teor. Geofiz. Akad. Nauk SSSR, 1, 95-115.

Panofsky, H. A., 1963: Determination of stress from wind and temperature measurements. Quart. J. Roy. Meteor. Soc., 89, 85-94.

Park, S.-J., S.-U. Park, C.-H. Ho, and L. Mahrt, 2009: Flux-gradient relationship of water vapor in the surface layer obtained from CASES-99 experiment. J. Geophys. Res., 114, D08115, doi:10.1029/2008JD011157.

Paulson, C. A., 1970: The mathematical representation of wind speed and temperature profiles in the unstable atmospheric surface layer. J. Appl. Meteor., 9, 857-861.

Poulos, G. S., and Coauthors, 2002: CASES-99: A comprehensive investigation of the stable nocturnal boundary layer. Bull. Amer. Meteor. Soc., 83, 555-581.

Shin, H. H., and S. Hong, 2011: Intercomparison of planetary boundary-layer parametrizations in the WRF model for a single day from CASES-99. Bound.-Layer Meteor., 139, 1-21.

Skamarock, W. C., and Coauthors, 2008: A description of the advanced research WRF version 3. Tech. Rep. TN-475+STR, NCAR, 113 pp.

Sorbjan, Z., 2010: Gradient-based scales and similarity laws in the stable boundary layer. Quart. J. Roy. Meteor. Soc., 136, 1243 1254.

—_, and A. A. Grachev, 2010: An evaluation of the flux-gradient relationship in the stable boundary layer. Bound.-Layer Meteor., 135, 385-405.

Stull, R. B., 1988: An Introduction to Boundary Layer Meteorology. Kluwer Academic, 666 pp.

van Ulden, A. P., and A. A. M. Holtslag, 1985: Estimation of atmospheric boundary layer parameters for diffusion applications. J. Climate Appl. Meteor., 24, 1196-1207.

Webb, K., 1970: Profile relationships: The log-linear range, and extension to strong stability. Quart. J. Roy. Meteor. Soc., 96, 67-90.

Weisman, M. L., C. Davis, W. Wang, K. W. Manning, and J. B. Klemp, 2008: Experiences with 0-36-h explicit convective forecast with the WRF-ARW model. Wea. Forecasting, 23, 407-437.

Wilson, D. K., 2001: An alternative function for the wind and temperature gradients in unstable surface layers. Bound. Layer Meteor., 99, 492-501.

Zhang, D. L., and R. A. Anthes, 1982: A high-resolution model of the planetary boundary layer-Sensitivity tests and comparisons with SESAME-79 data. J. Appl. Meteor., 21, 1594-1609. 